\title{
Far- and Extreme-UV Solar Spectral Irradiance and Radiance from Simplified Atmospheric Physical Models
}

\author{
J.M. Fontenla $\cdot$ E. Landi $\cdot$ M. Snow $\cdot$ T. Woods
}

Received: 23 August 2013 / Accepted: 8 October 2013 / Published online: 15 November 2013

(C) The Author(s) 2013. This article is published with open access at Springerlink.com

\begin{abstract}
This article describes an update of the physical models that we use to reconstruct the FUV and EUV irradiance spectra and the radiance spectra of the features that an given point in time may cover the solar disk depending on the state of solar activity. The present update introduces important modifications to the chromosphere-corona transition region of all models. Also, the update introduces improved and extended atomic data. By these changes, the agreement of the computed and observed spectra is largely improved in many EUV lines important for the modeling of the Earth's upper atmosphere. This article describes the improvements and shows detailed comparisons with EUV/FUV radiance and irradiance measurements. The solar spectral irradiance from these models at wavelengths longer than $\approx 200 \mathrm{~nm}$ is discussed in a separate article.
\end{abstract}

Keywords UV radiation · Transition region · Corona

\section{Introduction}

Spectra and images of the solar disk are used by Fontenla et al. (1999, 2009a, 2011, hereafter Articles 1,2, and 3, respectively) to construct a set of models for the characteristic features of the solar atmosphere present on the disk throughout the solar cycle. These features are

Solar Origins of Space Weather and Space Climate

Guest Editors: I. González Hernández, R. Komm, and A. Pevtsov

J.M. Fontenla $(\bowtie)$

NorthWest Research Associates Inc., Boulder, CO, USA

e-mail: jfontenla@nwra.com

E. Landi

Department of Atmospheric, Oceanic and Space Sciences, University of Michigan, Ann Arbor, MI, USA

M. Snow · T. Woods

Laboratory for Atmospheric and Space Physics, University of Colorado, Boulder, CO, USA 
described by models of their physical atmospheric structures that produce radiance (i.e. emergent intensity) spectra that agree with the available observations. The complete radiance spectra (currently $0.1 \mathrm{~nm}$ to 300 micron) from each type of structure are then weighted using solar images and added to yield the total solar radiation in the direction of Earth, and thereby the solar spectral irradiance (SSI) at $1 \mathrm{AU}$. The spectra are computed at variable, extremely high, spectral resolution; they contain at least ten points covering every spectral line. The spectral resolution in narrow lines reaches $\Delta \lambda / \lambda \approx 2 \times 10^{-6}$, and in the continuum a lower resolution is used. In order to compare our computations with available observations, the computed spectra are degraded to match the resolution of the observations with various instruments. For the calculations, the Solar Radiation Physical Modeling (SRPM: Fontenla, Balasubramaniam, and Harder, 2007) set of tools is used.

This article compares calculations with key spectral features observed from space and shows that the present simple (one-dimensional, steady) models of mid-resolution areas of the solar surface, $\approx 2 \times 2 \operatorname{arcsec}^{2}$, provide a fairly good representation of the SSI. Fine details of the spatial structure, e.g. loop structures, or of the spectral-line profiles often involve velocities that cannot be explained by these simple models. These details are important for understanding the internal physical structure of each individual feature but are not yet well understood. However, these details are of little importance for the applications to the Earth's upper-atmosphere modeling. The models presented here are a useful approximation to the averaging of the emission over those relatively small patches that are frequently observed in images of the full solar disk.

Our current scheme for determining the features present on the solar disk uses UV images from the Atmospheric Imaging Assembly (AIA) instrument onboard the Solar Dynamics Observatory (SDO) at several wavelengths. However, Solar Heliospheric Observatory (SOHO) images from the Extreme ultraviolet Imaging Telescope (EIT) instrument can easily be used as we did in the past, and Solar TErrestrial RElationship Observatory (STEREO) Extreme Ultraviolet Imager (EUVI) can also be used. For historical reconstructions, the Ca II K images can be used, although these do not directly provide information on the high-temperature components responsible for the UV and thus require some speculative assessment. We currently use both chromospheric and coronal images to discriminate between the features on the disk and be able to assign these features to atmospheric models. When the near-UV (NUV) and visible continuum are relevant, one should also use photospheric images to discriminate sunspot umbrae and penumbrae. Regarding sunspots, the distinction of, at least, these two features is very important because they have very different spectra. Also, the relative extent of umbra and penumbra are not well correlated and individual observations are necessary to determine their areas. However, in the present article we will not study sunspots because they are of little relevance for the range of wavelengths studied here.

Table 1 shows the indices of the models that we consider in the present as in Article 3 and partially in previous articles, the letter designations correspond to the solar features and a summary description of the feature is given. In this table sunspot models are omitted.

The behavior of the models temperature $v s$. height for layers below the chromospherecorona transition region are not significantly changed from those given by Article 3, except to smooth out some irregularities. However, the addition of more species and levels produced some differences in the electron density and thereby in the density stratification that is computed, as in Article 3, using hydrostatic pressure equilibrium with the same additional acceleration term as in that article. This additional term is an upward acceleration that increases the height-scale for better matching the observations at the limb.

In the transition region and corona, the improvements in the atomic data along with an improved treatment of the energy balance introduce important changes in the temperature vs. height. The use of CHIANTI 7.1 (Landi et al., 2013) electron-collision excitation 
Table 1 Current models for the solar disk features.

\begin{tabular}{llll}
\hline Feature & Description & $\begin{array}{l}\text { Photos.-chromos. } \\
\text { model index }\end{array}$ & $\begin{array}{l}\text { Corona } \\
\text { model index }\end{array}$ \\
\hline A & Dark quiet-Sun inter-network & 1300 & 1310 \\
B & Quiet-Sun inter-network & 1301 & 1311 \\
D & Quiet-Sun network lane & 1302 & 1312 \\
F & Enhanced network & 1303 & 1313 \\
H & Plage (that is not facula) & 1304 & 1314 \\
P & Facula (i.e. very bright plage) & 1305 & 1315 \\
Q & Hot Facula & 1308 & 1318 \\
\hline
\end{tabular}

and ionization rates largely improves the agreement with observations of many computed lines.

In the following, these changes are described and the resulting EUV/FUV spectra are compared with available observations of irradiance and radiance. UV spectral irradiance data are provided by the Solar Stellar Irradiance Comparison Experiment (SOLSTICE) instrument onboard the Solar Radiation and Climate Experiment (SORCE) satellite, and by the EUV Variability Experiment (EVE) instrument onboard the SDO satellite.

\section{The Non-LTE Computations and Atomic Data}

In the current calculation we have increased the number of levels for which full-NLTE is computed in detail and included more recent atomic data for many species. The improvements for the low ionization species, e.g. C, N, O, Si, Mg, at ionization stages II and III, now including collisional excitation data from CHIANTI 7.1, are more accurate than those used in Article 3 and earlier, which were derived according to the Seaton (1962) approximation. For several neutral species, we are now considering more levels. As a consequence of the increased number of levels, many lines that were not previously computed are now present in our spectrum.

For 198 higher ionization species, more levels and lines are now included in CHIANTI version 7.1 data. The complete data for the levels and transitions (with the only exception of proton collisional excitation) from this source have been used in the computation of the level populations using the effectively thin approximation. This approximation assumes that for most directions and important wavelengths the lines are optically thin, although for some paths (e.g. very close to the limb) this may not be the case. Also, this approximation assumes no optical pumping or interaction between transitions from different species, and is described in Article 3. Therefore, the effectively optically thin approximation in this article solves simultaneously the statistical equilibrium of all the level populations for each species at each height by including collisional rates and neglecting radiative rates other than spontaneous decay rates. The SRPM codes allow for including in the effectively thin approximation the radiative-transition rates driven by a prescribed illumination, e.g. external illumination, but these are not important for the layers and species considered here in which collisional transition and spontaneous-decay rates dominate. Also, the ionization of these species is computed according to the CHIANTI 7.1 data and procedure.

Table 2 shows the number of levels for the species computed here in full NLTE. These full NLTE calculations include low ionization stages in which it is estimated that the effectively 
Table 2 Species currently computed with full NLTE radiative transfer.
* Indicates species that were computed using the effective optically thin approximation. Higher ionization of these and other elements was also computed using the CHIANTI 7.1 data.

\begin{tabular}{llccl}
\hline Element & Abundance & $\begin{array}{l}\text { Ion I } \\
\text { levels }\end{array}$ & $\begin{array}{l}\text { Ion II } \\
\text { levels }\end{array}$ & $\begin{array}{l}\text { Ion III } \\
\text { levels }\end{array}$ \\
\hline $\mathrm{H}$ & 1.0 & 25 & - & - \\
$\mathrm{He}$ & 0.1 & 20 & 15 & - \\
$\mathrm{C}$ & $2.4 \times 10^{-4}$ & 45 & 27 & $*$ \\
$\mathrm{~N}$ & $0.9 \times 10^{-4}$ & 26 & 33 & $*$ \\
$\mathrm{O}$ & $3.9 \times 10^{-4}$ & 23 & 31 & $*$ \\
$\mathrm{Ne}$ & $6.92 \times 10^{-5}$ & 80 & 57 & $*$ \\
$\mathrm{Na}$ & $1.48 \times 10^{-6}$ & 22 & 14 & $*$ \\
$\mathrm{Mg}$ & $3.39 \times 10^{-5}$ & 26 & 14 & 54 \\
$\mathrm{Al}$ & $2.34 \times 10^{-6}$ & 18 & 14 & 32 \\
$\mathrm{Si}$ & $3.24 \times 10^{-5}$ & 35 & 14 & 60 \\
$\mathrm{~S}$ & $6.92 \times 10^{-6}$ & 20 & 30 & $*$ \\
$\mathrm{Ar}$ & $1.52 \times 10^{-6}$ & 48 & 57 & $*$ \\
$\mathrm{~K}$ & $1.20 \times 10^{-7}$ & 10 & $*$ & $*$ \\
$\mathrm{Ca}$ & $2.04 \times 10^{-6}$ & 22 & 24 & 34 \\
$\mathrm{Ti}$ & $7.94 \times 10^{-8}$ & 116 & 78 & 43 \\
$\mathrm{~V}$ & $1.00 \times 10^{-8}$ & 120 & 41 & 40 \\
$\mathrm{Cr}$ & $4.36 \times 10^{-7}$ & 102 & 34 & 20 \\
$\mathrm{Mn}$ & $2.45 \times 10^{-7}$ & 85 & 40 & 28 \\
$\mathrm{Fe}$ & $2.82 \times 10^{-5}$ & 119 & 120 & 90 \\
$\mathrm{Co}$ & $8.32 \times 10^{-8}$ & 65 & 28 & 50 \\
$\mathrm{Ni}$ & $1.70 \times 10^{-6}$ & 61 & 28 & 40 \\
\hline
\end{tabular}

thin approximation would not be accurate. Some species only become ionized to stages higher than II (i.e. charge more than one) within the lower transition region and therefore for them the effectively thin approximation is sufficient. This is the case of C III, N III, O III, and $\mathrm{S}$ III, which are now calculated in the effectively thin approximation but were computed in full-NLTE in previous articles.

Elemental ionization is calculated in full NLTE for the species as indicated in Table 2 with a valid number of levels. For these species, radiative interaction is expected to occur and the optically thin approach is not accurate. For the species where a valid number of levels in Table 2 is not specified, the effectively thin approximation using data in CHIANTI version 7.1 is used.

Collision rates from CHIANTI 7.1 were used, when available, for many species listed in Table 2 and all those not listed in the table and those not listed there. For the transitions not listed in CHIANTI the Seaton approximation was used to determine the collision excitation rates. In some cases, e.g. C I, N I, O I, and S I, some data were taken from those available in the literature as indicated in Article 3 and earlier articles. Collisional excitation by proton data are available for many species in CHIANTI 7.1 but after checking some cases it was found that its importance is secondary, at least in the tested cases.

Another improvement in the present calculations was the change of the photo-ionization cross-section for the first excited level of S I. This cross-section was previously assumed to be rather large from extrapolation of TOPBASE data (Seaton, 1987). However, we now estimate it to be smaller and adopted $6.0 \times 10^{-18} \mathrm{~cm}^{2}$ for its value at the head. The new value solves the issue with the computed edge at $\approx 134.55 \mathrm{~nm}$, which was overestimated before. 
There are still some issues with other FUV continua with edges at wavelengths longer than that of the Ly continuum. These issues are discussed in Section 5 below.

The calculations are carried out using the Solar Radiation Physical Modeling tools, now in version 2.2 (SRPMv2.2). These tools allow considering height-dependent abundance variations; however, our present calculations show no need to impose such variations. The present models use the same abundances throughout the photosphere/chromosphere/ transition-region/low-corona and produce good results for the SSI reconstruction. Effects of uncertainties in ionization, e.g. due to velocities, or in atomic data or atmospheric models, can very well explain some particular lines that may not be well represented in our results. Of course abundance variations are to be expected above the layers that we consider because mixing may not be very effective there, e.g. within the solar wind depending on its acceleration mechanism. In this article we use the abundance values listed in Table 2, which are the same as in Article 3.

For the species with a valid number of levels in Table 2 the calculations were carried out assuming that the indicated levels contain sublevels of different quantum number $J$. The sublevels are assumed to be populated in LTE within each level. The transitions between levels are computed considering the fully resolved fine structure of the lines that compose these transitions.

The collisional data used to calculate $\mathrm{C}$ I non-LTE were taken from the recent calculations by Wang, Zatsarinny, and Bartschat (2013). The authors provided collision strengths for the lowest 22 levels in LS coupling, which correspond to 42 fine-structure levels (or sublevels as in the paragraph above). Given the much larger atomic model, the calculations by Wang, Zatsarinny, and Bartschat (2013) are likely more accurate than the earlier calculations using a smaller atomic model. Comparison with earlier calculations and with other calculations by the authors with more limited atomic models show differences. For the transitions involving levels above those computed by Wang et al., we use collision rates following the Seaton formula. $\mathrm{C}$ I is a particularly complicated atom with many significant lines from high levels, and our calculations include 87 sublevels. However, these new C I data were not yet fully considered for calculating the autoionization and dielectronic recombination of $\mathrm{C}$, which is also importantly affected by the Lyman continuum and Ly $\alpha$.

\section{Structure of the Transition Region and Energy Balance}

The updates of the atomic data mentioned in the previous section largely improve the comparison with observations of lines formed at temperatures $T<10^{5} \mathrm{~K}$, and lines formed at, and above, $T=10^{6} \mathrm{~K}$.

Transition-region lines formed at temperatures in the range $10^{5}<T<10^{6} \mathrm{~K}$, e.g. of $\mathrm{O}$ IV and V and N IV and V in the range 40-80 nm, were overestimated by Article 3 . The improvements in atomic data mentioned in Section 2 do not solve this issue, but additional considerations are needed. If the same energy-balance scheme of Article 3 were used, these transition-region lines would be overestimated by factors ranging from two to ten.

These lines show that the energy-balance transition-region calculation used in Article 3 produces too smooth a temperature increase with height. The transition region computed in Article 3 uses the Fontenla et al. (1990, 1991, 1993, hereafter FAL 1, 2, and 3, respectively) formulation with a unit filling factor and considers the Spitzer thermal conductivity of electrons, in addition to the energy transported by particle diffusion described in FAL which is negligible in the temperature range $T \geq 10^{5}$ but important at lower temperatures. This calculation yields too large an energy downflow, which the radiative losses from the 
observed spectrum cannot dissipate. The Spitzer (1962) electron heat conduction in the transition region at temperatures above $\approx 10^{5} \mathrm{~K}$ leads to very large emission compared with the observations.

Since the spectrum calculated assuming energy balance and using Spitzer conductivity is already larger that the observed in the temperature range $10^{5}-10^{6} \mathrm{~K}$, but it matches the observed outside this range, any additional mechanical energy dissipation (while maintaining the Spitzer conductivity) would only produce even larger emission. Such larger emission would be required to maintain energy balance but would worsen the comparison of computations with observations.

Considering a filling-factor smaller than one leads to underestimation of the line emissions produced at temperatures below $10^{5} \mathrm{~K}$. Only an extremely sharp change in the filling factor at $T \approx 10^{5} \mathrm{~K}$, from a value near unity in the part of the transition region below to a small value above, could reconcile the theory with the observations. We do not know of any observational evidence indicating such an abrupt change in filling factor within the transition region.

We found an explanation that is physically consistent with the observations and with energy balance in the transition region that consists in a reduction of the heat conduction from the value given by the Spitzer formula. Such a reduction was observed in many laboratory experiments, e.g. Brysk, Campbell, and Hammerling (1975), and was named "flux limiting". We considered the flux-limiting issues and found that these are relevant to the chromosphere-corona transition region for most of our models although of course the parameters of the plasma are not the same as in these laboratory experiments. Flux-limiting occurs in laboratory experiments when the fast electrons that supply the Spitzer formula electron conductivity cannot carry sufficient energy for energy balance, or when the Knudsen number describing the ratio of their free-path to the characteristic distance of temperature variation is not small enough. Note that these two reasons are just different ways of looking at the same issue of physical constraints on the applicability of the Spitzer formula. These conditions can easily occur in the solar atmosphere due to the low electron densities in the corona and the very steep temperature gradient in the chromosphere-corona transition region that the observations indicate.

Although improvements in the atomic data and in the ionization equilibrium may still be needed and elemental abundance variations may exist, only the reduction of the electronconduction heat flux can solve the issue presented by all emission lines in the uppertransition-region and still remain consistent with those at lower temperatures. Such a reduction is needed to match the observed spectra and is also justified by basic physical considerations that are similar to those found in laboratory experiments.

In the quiet-Sun models the temperature gradient, and hence the heat flux, is smaller than in active regions but the density is also lower and therefore the mean free path of electrons becomes large. In the active-region models, the increased density shortens the mean free path but the temperature gradient is also larger. The physically consistent behavior resulting from consideration of flux limiting can only be described by a global calculation of the electron distribution function in the transition region (e.g. MacNeice, Fontenla, and Ljepojevic, 1991) but requires knowledge of the distribution function in the corona, which is likely non-Maxwellian. In principle, this distribution could be computed by taking into account the processes that supply the electron energy in the corona and sinks due to local dissipation and transport. Work on thermal conduction has been carried out in the context of the laboratory target preheating in laser-driven target implosions by Schurtz, Nicolai, and Busquet (2000) and references therein, and in the context of the solar wind by Bale et al. (2013) and references therein. An in-depth discussion of the validity of the Spitzer conductivity flux-limiting approaches has been given by Catto and Grinneback (2000). 
Because a global calculation is outside the scope of the present article, and because we do not know the electron distribution function in the corona, which determines the downward transport through the transition region, we use here the following ad-hoc formula, which produces the physical asymptotic limits for very large and very small temperature gradients and is similar to that suggested by flux-limiting considerations:

$$
K_{\text {eff }}=K_{\mathrm{a}}+K_{\mathrm{i}}+K_{\mathrm{SH}} /\left(1+\alpha F\left(T, n_{\mathrm{e}}\right) Z_{T}\right),
$$

where $K_{\text {eff }}$ is the effective energy transport coefficient that multiplied by the logarithmic gradient of the temperature $\left[\mathrm{Z}_{T}\right]$ gives the total heat flux. $K_{\mathrm{a}}$ and $K_{\mathrm{i}}$ give the heat-flux coefficient due to atoms conduction and diffusion carrying of ionization energy (see FAL articles), and $K_{\mathrm{SH}}$ is the electron conductivity given by the Spitzer (1962) formula, $\alpha$ is a constant coefficient, and $F\left(T, n_{\mathrm{e}}\right)$ is the following function:

$$
F\left(T, n_{\mathrm{e}}\right)=2.7 \times 10^{6} T^{2} n_{\mathrm{e}}^{-1} ;
$$

all quantities are in cgs units. In this formula, the term $\alpha F Z_{T}$, where $Z_{T}$ is the magnitude of the temperature gradient, parametrically describes the global issues. There are no compelling physical reasons for choosing the parametric form in Equation (2) over others, neither is there any good physical reason for picking one value of the parameter $\alpha$ over another value. We tried different values of $\alpha$ and concluded that a value of 7.0 produces the best agreement between the computed and observed line intensities. Thus, the parametric form and the value of the parameter $\alpha$ are defined empirically by matching the observations, but we note that the adopted function $F$ is proportional to the Knudsen number.

There are further complications in the calculations arising from two issues. The first issue is that the flux-limiting mentioned above should only be applied to the electron conduction using the Spitzer formula, but it should not affect the $\mathrm{H}$ and $\mathrm{He}$ diffusion and therefore does not affect the ionization energy transport (i.e. the reactive energy transport; see FAL articles about these other transport processes). When flux limiting is applied to the electron conduction, the relative importance of this reactive transport becomes even larger. This complicates the calculations at the lower temperatures and we address it by an iterative procedure. Because of uncertainty in ionized He diffusion, here we just established the lower-boundary condition of our transition-region energy balance arbitrarily at $T \approx 6 \times 10^{4} \mathrm{~K}$.

The second issue in the calculation is that a too large a flux-limiting function $[F]$ can lead to a situation in which even an infinite (or extremely large) temperature gradient at one layer could be insufficient to provide for the energy dissipated below. We have used an ad-hoc approach to deal with this problem, which arises at high temperatures because our computed value for $F$ increases steeply with increasing temperature and decreasing density. Of course, this issue is not a physical problem for energy transport in a general case, but only a problem for the flux-limiting approach. In reality, the heat flux could become highly nonlocal and only dependent on the global temperature differences but not on the temperature gradient at a particular location. In the present article we use an ad-hoc modification to the function $F$ to avoid this problem.

Table 3a gives the values of logarithmic temperature gradient, free-streaming heat flux (see Wyndham et al., 1982), Spitzer-formula heat flux, and the flux-limited heat flux in our calculation for each model at the layer of $T=2 \times 10^{5} \mathrm{~K}$. Most of the heat flux shown in that table produces emission by the lower part of the transition region and only a part of this flux reaches the region where the Ly $\alpha$ line center is formed. In our calculations for all models the flux-limited heat flux is about 0.18 of that given by the Spitzer formula, corresponding to $\alpha \mathrm{FZ}_{T} \approx 4.4$. This is a result of the calculations and was not imposed.

Figure 1 shows the effect on the temperature structure of the lower part of the transition region of the model for feature B (network cell interior, see Table 1) using different values 
Figure 1 The lower part of the transition region in model 1301, see Table 1, calculated using flux-limiting coefficient $[\alpha]$. The value 0 of the coefficient corresponds to the strict Spitzer formula for the electron conduction. different values of the

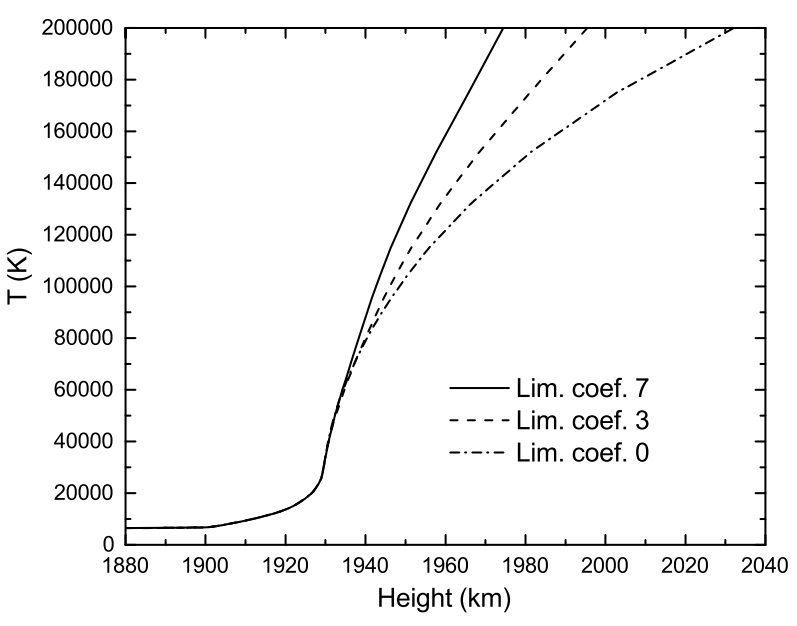

Figure 2 The lower part of the transition region for all models of the set indicated in Table 1, left to right models 1308, 1305, 1304, 1303, 1302, 1301, 1300.

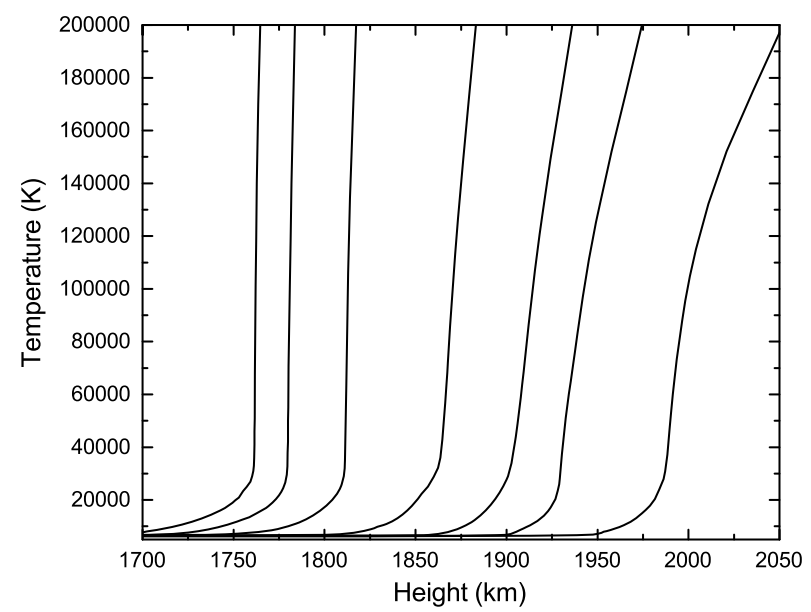

of the flux-limiting parameter $[\alpha]$. As the figure shows, increasing this parameter produces a steepening of the transition region, which does not affect the lower temperatures below say $8 \times 10^{4} \mathrm{~K}$. The steepening effect also occurs in the upper part of the transition region (not shown in this figure) and significantly affects many lines throughout the spectrum bringing the calculated lines closer to the observed, e.g. the Ne VI $40.193 \mathrm{~nm}$.

Note that, as the flux limiting becomes important, the total energy dissipated in the transition region by the electron heat-conduction decreases because the extension of this region decreases. The temperature gradient becomes stronger even when the heat flux decreases with respect to the non-flux-limited situation. All of these changes reduce the line emission when the flux-limiting parameter is increased. In this way flux limiting corrects the overestimation of the transition-region lines, bringing the calculations to better agreement with the observations than those of Article 3.

Figure 2 shows our final lower transition-region models that use limiting factor $\alpha=7.0$. These are adopted in the remainder of this article for the various models. Tables $3 \mathrm{a}$ and $3 \mathrm{~b}$ show the parameters in the current models for the point where the lower-layers models (photosphere/chromosphere/lower-transition-region) join the upper transition-region/low- 
Table 3a Temperature gradient, heat flux at $2 \times 10^{5} \mathrm{~K}$, and radiative flux above this level.

\begin{tabular}{llllll}
\hline Model & $\begin{array}{l}\mathrm{grad} \ln T \\
{\left[\mathrm{~cm}^{-1}\right]}\end{array}$ & $\begin{array}{l}F_{\mathrm{lim}} \\
{\left[\mathrm{erg} \mathrm{cm}^{-2} \mathrm{~s}^{-1}\right]}\end{array}$ & $\begin{array}{l}-F_{\mathrm{s}} \\
{\left[\mathrm{erg} \mathrm{cm}^{-2} \mathrm{~s}^{-1}\right]}\end{array}$ & $\begin{array}{l}-F_{\mathrm{h}} \\
{\left[\mathrm{erg} \mathrm{cm}^{-2} \mathrm{~s}^{-1}\right]}\end{array}$ & $\begin{array}{l}F_{\mathrm{r}} \\
{\left[\mathrm{erg} \mathrm{cm}^{-2} \mathrm{~s}^{-1}\right]}\end{array}$ \\
\hline $13 \times 0$ & $6.0 \times 10^{-8}$ & $1.0 \times 10^{7}$ & $2.2 \times 10^{5}$ & $4.0 \times 10^{4}$ & $8.4 \times 10^{4}$ \\
$13 \times 1$ & $1.1 \times 10^{-7}$ & $1.7 \times 10^{7}$ & $3.8 \times 10^{5}$ & $7.0 \times 10^{4}$ & $1.7 \times 10^{5}$ \\
$13 \times 2$ & $1.3 \times 10^{-7}$ & $2.6 \times 10^{7}$ & $4.7 \times 10^{5}$ & $9.0 \times 10^{4}$ & $3.1 \times 10^{5}$ \\
$13 \times 3$ & $2.7 \times 10^{-7}$ & $4.4 \times 10^{7}$ & $9.6 \times 10^{5}$ & $1.5 \times 10^{5}$ & $7.1 \times 10^{5}$ \\
$13 \times 4$ & $5.1 \times 10^{-7}$ & $8.7 \times 10^{7}$ & $1.8 \times 10^{6}$ & $3.4 \times 10^{5}$ & $2.2 \times 10^{6}$ \\
$13 \times 5$ & $8.6 \times 10^{-7}$ & $1.4 \times 10^{8}$ & $3.1 \times 10^{6}$ & $5.8 \times 10^{5}$ & $4.1 \times 10^{6}$ \\
$13 \times 8$ & $1.2 \times 10^{-6}$ & $2.0 \times 10^{8}$ & $4.3 \times 10^{6}$ & $8.0 \times 10^{5}$ & $6.0 \times 10^{6}$ \\
\hline
\end{tabular}

Table 3b Other parameters at $2 \times 10^{5} \mathrm{~K}$.

\begin{tabular}{llllll}
\hline Model & $\begin{array}{l}p \\
{[\text { dyne cm }}\end{array}$ & $\begin{array}{l}\left.n_{\mathrm{e}}\right] \\
{\left[\mathrm{cm}^{-3}\right]}\end{array}$ & $\begin{array}{l}q_{\mathrm{r}} \\
{\left[\mathrm{erg} \mathrm{cm}^{-3} \mathrm{~s}^{-1}\right]}\end{array}$ & $\begin{array}{l}q_{\mathrm{m}} \\
{\left[\mathrm{erg} \mathrm{cm}^{-3} \mathrm{~s}^{-1}\right]}\end{array}$ & $\begin{array}{l}\text { DEM } \\
{\left[\mathrm{cm}^{-5} \mathrm{~K}^{-1}\right]}\end{array}$ \\
\hline $13 \times 0$ & 0.15 & $2.7 \times 10^{9}$ & $2.1 \times 10^{-3}$ & $1.8 \times 10^{-4}$ & $6.1 \times 10^{20}$ \\
$13 \times 1$ & 0.23 & $4.4 \times 10^{9}$ & $5.3 \times 10^{-3}$ & $5.2 \times 10^{-4}$ & $8.8 \times 10^{20}$ \\
$13 \times 2$ & 0.35 & $6.7 \times 10^{9}$ & $1.2 \times 10^{-2}$ & $1.2 \times 10^{-3}$ & $1.7 \times 10^{21}$ \\
$13 \times 3$ & 0.61 & $1.1 \times 10^{10}$ & $3.5 \times 10^{-2}$ & $3.5 \times 10^{-3}$ & $2.2 \times 10^{21}$ \\
$13 \times 4$ & 1.2 & $2.2 \times 10^{10}$ & $1.4 \times 10^{-1}$ & $1.4 \times 10^{-2}$ & $4.7 \times 10^{21}$ \\
$13 \times 5$ & 2.0 & $3.8 \times 10^{10}$ & $3.8 \times 10^{-1}$ & $3.7 \times 10^{-2}$ & $8.4 \times 10^{21}$ \\
$13 \times 8$ & 2.8 & $5.3 \times 10^{10}$ & $7.4 \times 10^{-1}$ & $7.4 \times 10^{-2}$ & $1.2 \times 10^{22}$ \\
\hline
\end{tabular}

corona models, namely the layer with $T=2 \times 10^{5} \mathrm{~K}$. The parameters listed are derived from energy balance through the lower transition region and applied as a starting point for the energy balance of the upper transition region. These parameters are for the final set of models that use flux-limiting coefficient value $\alpha=7.0$, which is the value that overall produces the best match between the calculations and observations of the spectra.

\section{Structure of the Low Corona}

The structure of the lower corona is similar to that in Article 3, however, the upper part of the transition region included in our models is now computed using the same flux-limiting formula and parameter $\alpha$ as for the lower part of the transition region. As before, we merge the temperature structure of the upper part of the transition region with an extended region at coronal temperatures that is shown in Figure 3.

In order to produce a smooth fit of the corona with the upper part of the transition region we use the following mechanical-heating formula:

$$
q_{\mathrm{m}}=10^{-22} n_{\mathrm{e}} n_{\mathrm{p}}\left(\frac{T}{10^{6}}\right),
$$

where $n_{\mathrm{e}}$ and $n_{\mathrm{p}}$ are the electron and proton densities, respectively. We do not believe that this formula describes theoretically the coronal heating, but this formula has the approximate properties needed. At $T=2 \times 10^{5} \mathrm{~K}$ the mechanical heating $\left[q_{\mathrm{m}}\right]$ is $\approx 0.1$ of the radiative 
Figure 3 The structure of the coronal models adopted for various features described in Table 1. Top to bottom the model indices are 1318, 1315, 1314, $1313,1312,1311$, and 1310 .

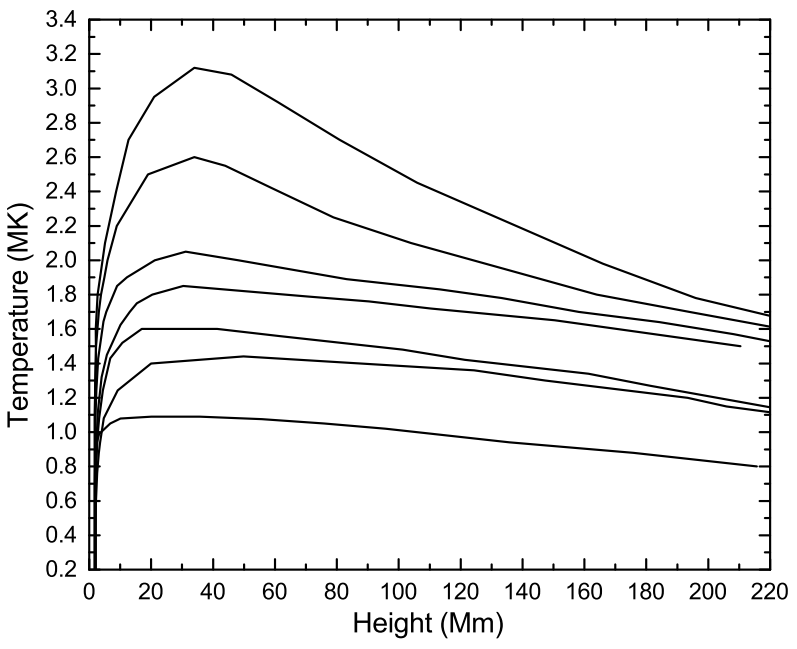

losses $\left[q_{\mathrm{r}}\right]$, and only at $T \approx 6 \times 10^{5} \mathrm{~K}$ does the mechanical heating balances the radiative losses for all models. The same formula is used for all models, but the amount of mechanical heating is different because of the different pressure and maximum temperature in each of them. We adopt coronal layers above and around the point where $q_{\mathrm{m}} \approx q_{\mathrm{r}}$ independently of this formula and with a smooth temperature maximum and decay above it.

These temperature maximum and decay are determined in order to match the observations the best that we can do it currently. Unfortunately, there are not many observations of active-region spectra with high spectral resolution and reliable absolute calibration. As new observations become available we hope that the layers around and above the temperature maximum will be better determined. However, statistics of many observations are needed because many differences occur between various active regions. In addition these regions have very inhomogeneous temperature and density due to their loop fine structure, and therefore comprehensive spatial coverage and a statistical description are needed. Individual loops detailed structure is highly variable in space and time and have been modeled by other authors using very strong simplifications (e.g. Rosner, Tucker, and Vaiana, 1978; Serio et al., 1981, also see review by Reale, 2010). Our models are focused on spectralirradiance synthesis and do not attempt to model individual active-region loops; instead the present models are intended to describe the SSI relevant properties of an ensemble of such loops, over the term of several hours, which is relevant to SSI and its effects on Earth's upper atmosphere.

An issue remains with relatively cool structures extending through and sometimes over the low corona, namely the more or less stable prominences (with very complicated internal fine structure), and also the short-lived high-speed jets. We do not model these because both pose problems of their own resulting from complex interactions between the plasma, which is sometimes but not fully ionized, and magnetic fields. In the present work we neglect these structures in the context of overall solar irradiance, although they could have effects at some wavelengths due to absorption of underlying coronal emission.

Model 1310 corresponds to somewhat cooler regions of the lower corona often associated with coronal holes in X-ray images. However, this model does not represent all that can be called coronal hole. Prominences cool overlying material described above, which absorb radiation from the underlying low corona and therefore would produce a void in the X-ray emission. Such a void can be partially due to the absence of coronal material in 
the threads that generally form a prominence. However, underneath it and in between the threads, coronal material may exist whose emission is obscured by the less ionized material in the threads. Since in the present work we are not considering the cool material embedded in the corona, we do not address these cases in the present article.

Models 1311, 1312, and 1313 correspond to the magnetic structure of quiet Sun, and are the extensions of their counterparts in the low transition region and chromosphere supergranular structure described by models 1301, 1302, and 1303 (see Table 1). It is implied in the construction of the models that the coronal features are built as an upwards extension of the chromospheric models, but it is apparent that in images corresponding to high temperatures that the supergranular structure cannot be identified. This may be simply due to the closing of the magnetic fields in relatively low-altitude loops that form a more or less uniform background. Our use of the set of models for spectral-irradiance reconstruction assumes that at coronal layers the model 1311 for feature B is representative of the mix. Another alternative we have tried is to assume that the components are mixed in the same proportion as their chromospheric counterparts and form a background of quiet Sun that can be somewhat enhanced if the fraction of the more active features, e.g. 1313, increases. The issue of whether the so-called quiet-Sun background changes during the solar cycle is complex because, even far away from the chromospheric active regions, so-called interconnecting coronal loops (which connect relatively remote active regions) may cover much of the solar disk at very high-activity times. These interconnecting loops have a contribution to the EUV spectral irradiance that can hardly be assigned to quiet-Sun structures. Depending on their brightness in EUV images, in a pixel we may assign them to one of our activeregion models (usually model 1314) but details on this image-processing issue are outside the scope of the present article.

Models 1314, 1315, and 1318 correspond to active regions. They are designed to approximately match the most common features of active regions outside of flares. Still, after the impulsive phase of flares, during flare decay a region on the disk may exhibit traits similar to some of these models. The three active-region models are characterized by different peak temperatures and also different pressures at the base (see Tables $3 \mathrm{a}$ and $3 \mathrm{~b}$ ).

Active regions are very heterogeneous and most likely all the components described by our models exist and are intermingled. However, for SSI modeling we use images to discriminate areas corresponding to one or another of the models because a mix would be very hard to assess from existing full-disk images. In the future, images with better spectral discrimination and calibration could improve this. Also, note that all of the models (when considering the entire chromospheric and coronal parts) contain a complete range of temperatures from the chromosphere up to a different maximum coronal temperature. This would correspond to a vertical continuity of the structure, different from observations showing curved loops; however, it can describe an ensemble of active-region compact loops. For high-altitude loops, the vertical structure is clear at different viewing angles and especially toward the limb. Depending on the observing angle these coronal loops may overlay regions that can or cannot be described as their loop footpoints. Because of this, we do not use the same identification of chromospheric for coronal features in the SSI reconstruction but instead compute the relative areas of the coronal components based on coronal images. The areas determined to be active regions from chromospheric or images of the lower part of the transition region (e.g. in He II $30.4 \mathrm{~nm}$ ) can overlap or have an offset from the coronal active regions.

For our computation of the spectra from the coronal features, we use spherical coordinates over patches corresponding to the observed areas of the features. Note that for the lower layers (photosphere, chromosphere, and lower transition region) the radial extent is 
so small that a plane-parallel approach is sufficient and breaks down only very close to the limb where the contribution to the irradiance is very small. However, a much larger limb brightening would result if we had not considered spherical coordinates and optical thickness effects due to the long path. These issues were verified by computing cases near the limb and comparing the computed center-to-limb variation with that observed in images from SDO/AIA.

One issue remains to discuss concerning the areas just above the limb. At many $\mathrm{XUV} / \mathrm{EUV}$ wavelengths these areas look bright in the observations, e.g. the well-known doubling of the intensity just above the limb. Detailed calculations using spherical coordinates show that for the quiet Sun their contribution to the irradiance is not very important. Our calculations match well the observed center-to-limb behavior near, at, and above the limb (except for the fainter regions well above the limb where instrumental effects are uncertain).

The consideration of the low corona just above the limb does not affect the irradiance for the quiet Sun. Active regions seen above the limb could contribute significantly when the disk is covered by quiet Sun. The result is just a temporal smoothing effect on the SSI rotational-modulation variations. When the active region comes into view its emission usually far surpasses that seen above the limb before, and when an active region disappears from the disk the change far surpasses that of its residual emission from above the limb. In any case, our methods could include the emission above the limb due to patches of active region by using three-dimensional radiative-transfer calculations if the horizontal extent of the active region is known or can be inferred from images.

Table $3 \mathrm{a}$ also shows the total radiative losses from the coronal models, i.e. the radiative flux $\left[F_{\mathrm{r}}\right]$ emitted by the upper-transition-region/low-corona of our models at $T>2 \times 10^{5} \mathrm{~K}$. These radiative fluxes are, for the coolest model, nearly twice the amount of downward energy flux by conduction, but become increasingly larger for the hotter models up to almost ten times the downward heat conduction. In terms of the emitted radiation this implies that the hot coronal lines emit more energy than the transition region for the cooler models and much more for the hotter models. The result in this table is different from what would occur if Spitzer conductivity were assumed, i.e. $\alpha=0$, because in that case the hotter models would have upper-transition-region/low-corona radiation comparable with the downward energy flux while the cooler models would have much less upper-transition-region/lowcorona radiation than downward energy flux. While the trends with increasing maximum coronal temperature are similar, the absolute values are quite different.

\section{EUV/FUV Continua Issues}

Here and in the following sections the SSI will be given in the usual units of $\mathrm{W} \mathrm{m}^{-2} \mathrm{~nm}^{-1}$, which is equivalent to $10^{2} \mathrm{erg} \mathrm{cm}^{-2} \mathrm{~s}^{-1} \AA^{-1}$.

The FUV continua computed from our models matches the observations at many wavelengths but not yet at all wavelengths. In the range $168-200 \mathrm{~nm}$ disagreement with observations remains. There are two other small ranges just short of $124 \mathrm{~nm}$ and of $50.4 \mathrm{~nm}$ where there is also some disagreement. The present section discusses these issues in detail for model 13x1 (resulting from combining chromospheric and coronal models 1301 and 1311), i.e. adding the spectrum from 1301 and 1311, but similar considerations apply to the other models of the set. Also, the same issue reflects on both the radiance and the solar spectral irradiance (SSI), and here we discuss the issue in both contexts.

Figure 4 shows a comparison of the computed and observed SSI for model $13 \times 1$. This figure shows three intervals of concern in the matching of the continuum. One of them is 
Figure 4 The overall SSI at $0.1 \mathrm{~nm}$ resolution computed for model $13 \times 1$ and the observations.

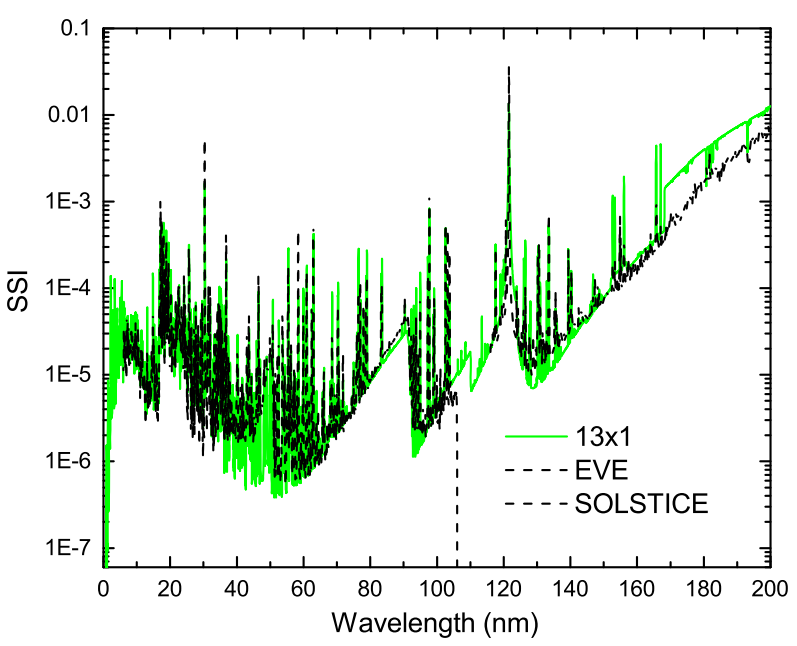

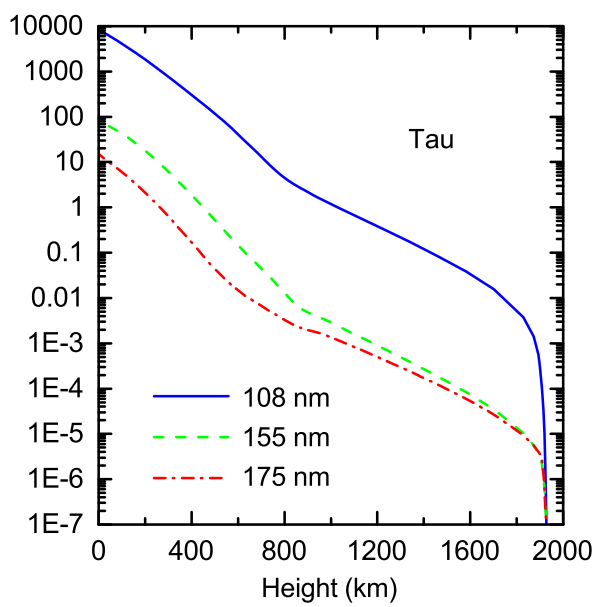

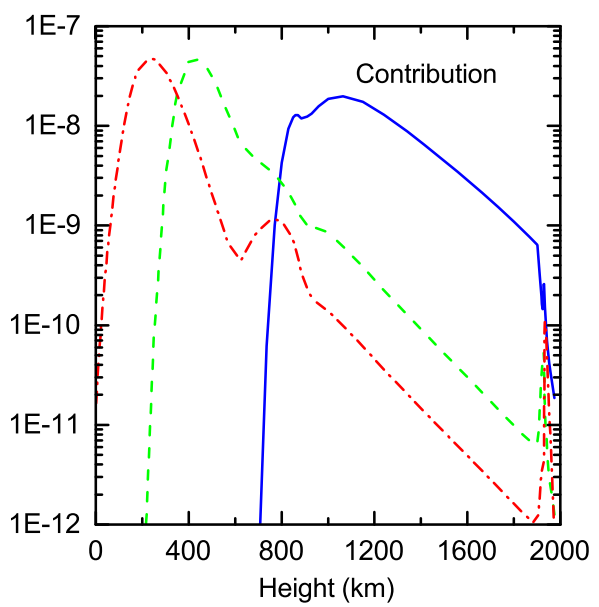

Figure 5 Optical depth (left) and "intensity contribution function" (right) at disk center as function of height for model 1301 at three selected continuum wavelengths.

wavelengths longer than $\approx 170 \mathrm{~nm}$, the others are small ranges below $124 \mathrm{~nm}$ and below $\approx 50 \mathrm{~nm}$. Details regarding the lines will be shown and discussed in Section 7; however, the continuum range just short of $124 \mathrm{~nm}$ is mixed up and interacts with the Ly $\alpha$ far wings.

Figure 5 shows the optical depth and the contribution functions for model 1301 at three wavelengths in the continuum. These wavelengths are selected to be representative of two of the issues mentioned above. For the purpose of this article the contribution function is redefined as the attenuated total emissivity (see Fontenla, Balasubramaniam, and Harder, 2007) but now divided by the emergent intensity. This change in the definition allows us to show this function at various wavelengths, with disparate emergent intensities, into the same figure and also ensures that for all curves the integral is unity. The emergent intensities at disk center for these wavelengths are: 457.3 for $175.0 \mathrm{~nm}, 297.1$ for $155.5 \mathrm{~nm}$, and 26.8 for $108.0 \mathrm{~nm}$. Here and in the following all emergent intensities are given in the usual units of $\operatorname{erg~cm}{ }^{-2} \mathrm{~s}^{-1} \AA^{-1} \mathrm{sr}^{-1}$, which is equivalent to $10^{-2} \mathrm{~W} \mathrm{~m}^{-2} \mathrm{~nm}^{-1} \mathrm{sr}^{-1}$, but 
see the detailed explanation about SSI and emitted intensity units in Fontenla et al. (1999) Appendix B.

A sharp edge occurs in the computed spectrum at the wavelength of the $\mathrm{Si}$ I continuum edge from the first excited state, namely $168.211 \mathrm{~nm}$. This atomic level has very low energy and is the lowest of the singlet, and at shorter wavelengths from the edge the intensity is smaller than at longer wavelengths from the edge. The ground level of Si I is a triplet level with an ionization edge at $152.096 \mathrm{~nm}$, and for this the intensity is a bit smaller at wavelengths longer than the edge than sorter from the edge, i.e. the opposite behavior to that of the $168.211 \mathrm{~nm}$ edge. Despite the correspondence to the ground level of Si I of the $152.0 \mathrm{~nm}$ edge, this jump is smaller than that of the $168.2 \mathrm{~nm}$ edge because the former is produced only a little bit above the temperature minimum. Instead, the $168.2 \mathrm{~nm}$ edge forms at a location of significant temperature gradient decreasing with increasing height. Another edge is shown in our calculation, but it is barely visible in Figure 6, at $198.596 \mathrm{~nm}$, which corresponds to the second excited state of $\mathrm{Si}$ I, which also belongs to the singlet. In summary, our calculation shows the Si I edges from all of the lower states. In stark contrast, the spectra observed by SOHO/SUMER (emitted intensity at disk center) or by SOLSTICE (SSI) do not show these edges. Instead they show no obvious jump in intensity but only slight changes in slope at $152.0 \mathrm{~nm}$. SUMER only covers up to $161.0 \mathrm{~nm}$ and cannot show the $168.2 \mathrm{~nm}$ edge, but SOLSTICE shows that at $168.0 \mathrm{~nm}$, again, only changes in slope occur and important absorption lines only occur at wavelengths longer than $\approx 180 \mathrm{~nm}$. The computed SSI spectra at wavelengths longer than the $168.2 \mathrm{~nm}$ edge show absorption lines and large continuum intensities that are absent in SOLSTICE spectra at wavelengths shorter than $200 \mathrm{~nm}$.

The reason for the behavior in our computation is shown by Figure 5. At $155.5 \mathrm{~nm}$ and other wavelengths shorter than $168.2 \mathrm{~nm}$, the continuum forms at higher altitude than that $175.0 \mathrm{~nm}$, and is formed in a region of the atmosphere with a small negative temperature gradient and at locations where the source function is affected by illumination from above and subject to NLTE effects. Instead, at $175.0 \mathrm{~nm}$ and other wavelengths longer than $168.2 \mathrm{~nm}$, the continuum forms at the top of the photosphere or within the lower-chromosphere where the negative temperature gradient is large and non-LTE effects are small. Therefore, we conclude that, within the lower chromosphere, at or below the temperature minimum, some important opacity sources are not included in our calculation but are present in the Sun. We speculate that the gradual behavior and lack of observed jumps or absorption lines, as well as the relatively large density and low temperatures in the layers involved, point to molecular photodissociation opacity that is not yet identified. It is remarkable that even at the $0.1 \mathrm{~nm}$ resolution of SORCE/SOLSTICE only few and weak fluctuations due to lines are observed, and that a few groups of lines that our calculation show in absorption around 180 are shown in emission by SOLSTICE, see Figure 4. This behavior is inconsistent with what could be inferred from Avrett and Loeser (2008) Figures 1 and 6 that predict very large fluctuations in intensity $v s$. wavelength in the range $170-200 \mathrm{~nm}$, which are not compared with observations in that article and seem to be incompatible with the SORCE/SOLSTICE $0.1 \mathrm{~nm}$ resolution data that we show in our Figure 4.

One of the problems for improving our understanding of this continuum is the lack of adequate computations of many molecular opacities in the FUV. Calculations exist for molecules in the interstellar medium, but these are not very useful for the solar atmosphere because of the substantial temperature and density of the low chromosphere affecting the populations of vibrational-rotational levels.

Let us now discuss the issues of the $C$ I edge just short of $124.0 \mathrm{~nm}$. The edge from the $\mathrm{C}$ I ground level occurs at $110.107 \mathrm{~nm}$, a triplet level, and that from the first excited state at $124.027 \mathrm{~nm}$, a singlet level. Both edges are clearly observable and our calculations overes- 
timate the latter. Figure 5 shows that the continuum at $108.0 \mathrm{~nm}$ is formed almost entirely in the upper chromosphere as C II recombines into C I. For the edge at 124.0, i.e. within the Ly $\alpha$ red wing, some contribution to the emission occurs from the temperature-minimum region where $\mathrm{C} \mathrm{I}$ is over-ionized by illumination from above. The issue with the overestimated C I edge arises from non-LTE effects and is more important here than in Article 3. As was explained in Section 2, we are now using a more recent calculation of the collisional excitation rates of $\mathrm{C} \mathrm{I}$, and these rates are generally larger than those used in Article 3. In particular the collision strength for the excitation from the ground level to the first excited is now about 100 times larger, at $T=5000 \mathrm{~K}$, and a larger fraction of $\mathrm{C}$ I is in the first excited state throughout the chromosphere. This larger population of level 2 produces a somewhat larger ionization of $\mathrm{C}$ I because of the photo-ionization produced by Ly $\alpha$ pumping, and enhances the $\mathrm{C}$ I ionization through the upper chromosphere and at the temperatureminimum region. As a result of this, the emitted $\mathrm{C}$ I continua is enhanced by recombination into both levels 2 and 1 . However, many of the photons emitted by recombination into level 1 are absorbed at larger heights leaving the strongest effect on the continua to level 2.

Our computed Ly $\alpha$ profile and corresponding non-LTE uses partial-frequency-redistribution and matches the observations at wavelengths longer than the $124 \mathrm{~nm}$ edge. The apparent enhancement of the Ly $\alpha$ line wings at wavelengths shorter than $124.0 \mathrm{~nm}$ results from the effects of the $\mathrm{C}$ I continuum. For C I non-LTE we use a discrete grid wavelength that accounts for the dependence of the $\mathrm{C}$ I ionization on Ly $\alpha$, and we verified that improving the grid by doubling the number of points in the quadrature used does not affect importantly our results. Therefore, we attribute most of the increased recombination to the excited level of $\mathrm{C}$ I in our present models, at layers around the temperature minimum, to the increased collision rate of the inter-combination transition 1-2 discussed above. Considering the complicated nature of the $\mathrm{C}$ I atom and the interaction with Ly $\alpha$, it is likely that further improvements in the collision rate, the $\mathrm{C}$ I ionization, or in the photo-ionization cross-sections from the $\mathrm{C}$ I levels could improve the agreement with the observations for the $124.0 \mathrm{~nm}$ continuum edge.

The third issue with the continuum is a minor one from the standpoint of SSI calculations. Article 3 indicated that the He I $50.4 \mathrm{~nm}$ continuum not shown in our calculations likely results from enhanced ionization of He I, i.e. increased presence of He II, at the top of the chromosphere due to illumination from the corona above. The observed slope of the He I continuum that can be discerned in between the coronal lines corresponds to a temperature of about $1.5 \times 10^{4} \mathrm{~K}$, which corresponds to the top of the chromosphere. At this temperature there would not be much He II unless coronal illumination produces additional ionization of He I. However, from the irradiance standpoint, the large coronal emission lines are much more important than the He II recombination continua around $50.4 \mathrm{~nm}$ and therefore this continuum does not pose a very important issue.

In the present calculation we have approximately solved the previous minor issue of the gap between the merging Ly lines and the continuum edge by extending the continuum up to the wavelength of the center of the first Ly line which we do not explicitly compute. Our current $\mathrm{H}$ atom includes 25 levels and therefore the extension of the continuum is carried up to the Ly line center corresponding to level 26. This mimics the merging of the Ly lines into the continuum solving some issues about SSI in the small gap that Article 3 showed. Although overall this is a very small gap it is significant because of its effects on the telluric lines.

The present computation of the dominant Si I continua spanning the $124-168 \mathrm{~nm}$ range matches well the radiance and irradiance observations. Also, the computation of the $\mathrm{H} \mathrm{I}$ continuum shorter than $\approx 91.3 \mathrm{~nm}$ matches well the observations of radiance and irradiance by SOHO/SUMER and SDO/EVE, respectively. 


\section{Comparison of Emitted Intensity (Radiance) with Observations}

In this section we compare the computed spectra with radiance (i.e. emitted intensity) observations at solar-disk center. The comparisons use some quiet-Sun atlases derived from the following instruments: Solar Ultraviolet Measurements of Emitted Radiation (SUMER: Wilhelm et al., 1995) onboard SOHO, EUV Imaging Spectrometer (EIS: Culhane et al., 2007) onboard Hinode, and the Coronal Diagnostic Spectrometer (CDS: Harrison et al., 1995) onboard SOHO. In all cases, we convolved the computed spectrum with the instrument resolution, which is known for the first two instruments but lesser understood for CDS. However, our profiles are computed for zero velocity and a "turbulent broadening velocity" that our models specify, and at the nominal vacuum wavelengths stated by NIST (Kramida et al., 2013). Random velocities in the Sun would cause the profiles to be somewhat more broadened, and systematic velocities would shift them, but we do not attempt here to correct for that.

It is, of course, impossible to show here the spectra in all their detail. The graphs shown in detail in this article only include several key spectral lines, and more complete comparisons over the whole ranges of the atlases are posted at www.galactitech.net/SRPMrel2013/. The computed spectral data are also available there at the native, variable sampling (as mentioned in Article 3, and in the introduction of this article; the resolution is higher over the lines than in the continuum and the computations correspond to snapshots of infinitesimal bandwidth). These computed spectra can be convolved with any instrument resolution taking into account any given bandpass or instrumental profile. For all comparisons shown in this article we have used a filter with bandpass shape given by the cosine square truncated at the first zeroes on either side of the central wavelength. This type of bandpass is designated here as $\cos ^{2}$, and the width of the bandpass is different when comparing our computations with different instruments. We consider this bandpass similar in some respects to a Gaussian but it does not require an arbitrary truncation, and it is also similar to the central part of a diffraction pattern. In the comparisons discussed here, only the full-width-at-half-maximum is given (hereafter FWHM), and we do not have reliable data on the various instruments' detailed profiles. We consider the $\cos ^{2}$ bandpass as likely close to the true instrumental profile. When convolution with the $\cos ^{2}$ bandpass is done, the sampling (i.e. output wavelength spacing) is $1 / 5$ of the FWHM nominally given. This sampling is often more closely spaced than the observational data.

In this section, we compare in detail important lines computed with the available quietSun atlases from various instruments. Because the radiance observations are nominally spatially resolved data representative of quiet Sun, the criteria for selecting quiet-Sun areas becomes relevant and may not have been identical in all cases. In the following figures we indicate as $13 \times 0,13 \times 1,13 \times 2,13 \times 3$ the additions of the coronal and chromospheric parts of the corresponding models (of course including the complete transition region). For instance, model $13 \times 1=1301+1311$ indicates the sum of the spectrum from models 1301 and 1311. This is done assuming that at disk center the coronal part is optically thin and therefore the procedure adopted corresponds to appending the coronal model to the top of the chromospheric model forming a continuous vertical structure.

\subsection{Comparison of Selected Lines with SOHO/SUMER}

The SOHO/SUMER quiet-Sun atlas by Curdt et al. (2001) was constructed from many observations during solar minimum and therefore probably represents a weighted average between the various features that we represent by models $13 \times 0,13 \times 1$, and $13 \times 2$, and maybe 

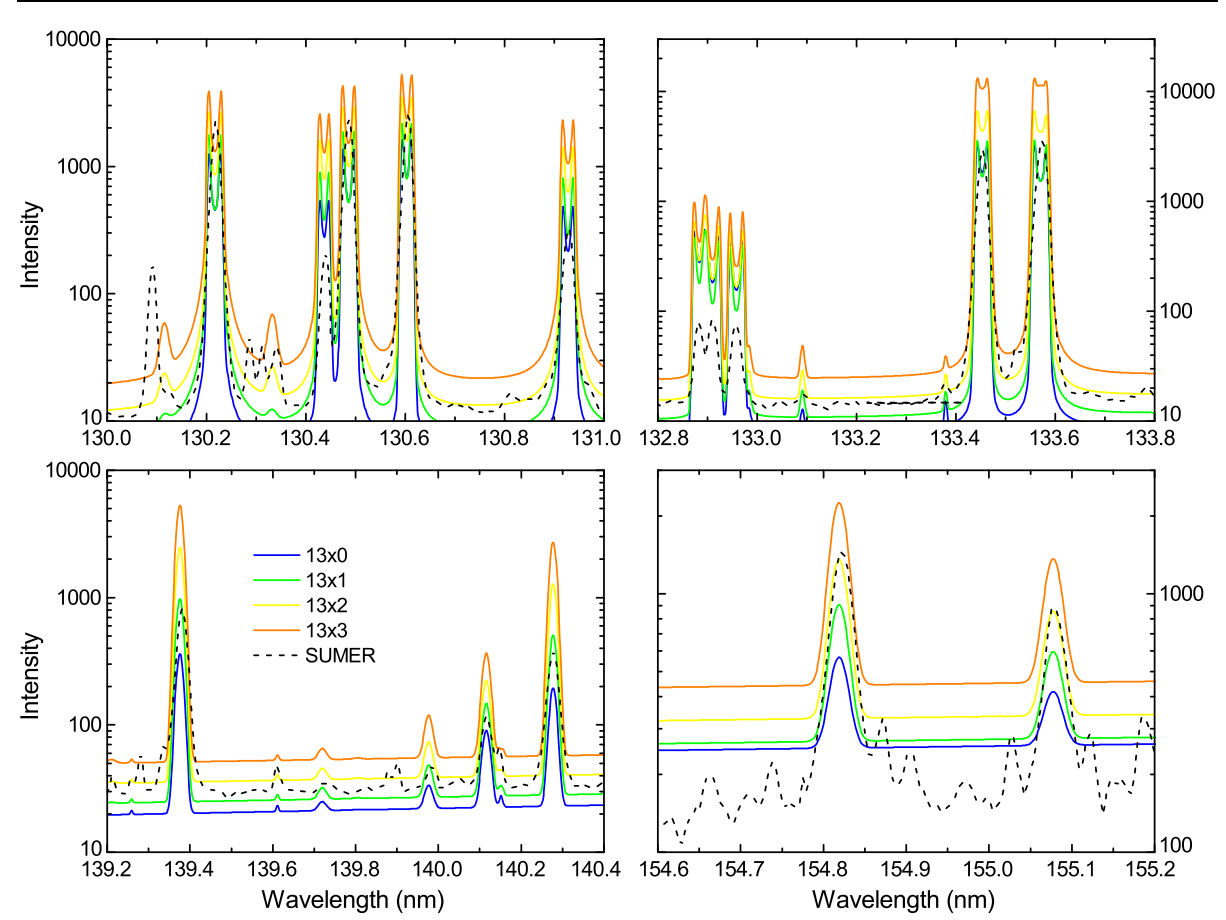

Figure 6 Top-left panel, the spectral range 130-131 nm showing the important lines of O I 130.217, 130.486, and $130.603 \mathrm{~nm}$, and also Si I 130.437 and $130.828 \mathrm{~nm}$. Top-right panel: the range $132.8-133.8 \mathrm{~nm}$ showing the important lines of C II 133.453, 133.566, and $133.571 \mathrm{~nm}$, and weak C I lines from 132.8 to almost $133.0 \mathrm{~nm}$. Bottom-left panel: the range 139.2 - 140.4 nm showing the important lines of Si IV 139.376, and $140.277 \mathrm{~nm}$. Bottom-right panel: the range $154.6-155.2 \mathrm{~nm}$ showing the important lines of C IV 154.819 and $155.077 \mathrm{~nm}$.

even a few percent of $13 \times 3$. The exact weights are not known to us although most likely the contribution from $13 \times 1$ is dominant and there is some sort of compensation between the brighter and fainter features. For this reason, in Figures 6 and 7 we compare the SUMER data with the three quiet-Sun components of our model set. For this comparison we convolve the computed spectra with a $\cos ^{2}$ bandpass of 5.5 pm (i.e. $55 \mathrm{m \AA}$ ) FWHM.

Figure 6 (top panels) shows comparisons of the most important chromospheric lines of $\mathrm{C}$ and $\mathrm{O}$ that were observed by SUMER. A good match is obtained for the $\mathrm{O}$ I and C II strong lines shown in the upper panels, although the computed profiles display a weak reversal which is not shown by SUMER. It has been argued that macroscopic velocities could eliminate this reversal, but we do not want to argue this because SUMER sampling of line profiles is not very good and because observations by other instruments may display such reversals. A group of C I lines in the range 132.8-133.0 nm, in the top-right panel, is overestimated and this is consistent with the overestimate of the $\mathrm{C}$ I continuum that was discussed in Section 5. Therefore, the question becomes one of how accurate the new C I collision-strength data are for these lines. The bottom-right panel shows some differences in the continuum longer than $152.0 \mathrm{~nm}$ that were discussed in Section 5.

Figure 6 (bottom panels) shows important low transition-region lines of Si IV and C IV. The C IV lines are somewhat underestimated by the calculations shown here that use a flux-limiting coefficient of 7.0 and are better represented with a coefficient of 3.0; they are overestimated by assuming that the coefficient is equal to 0 (i.e. considering the full 

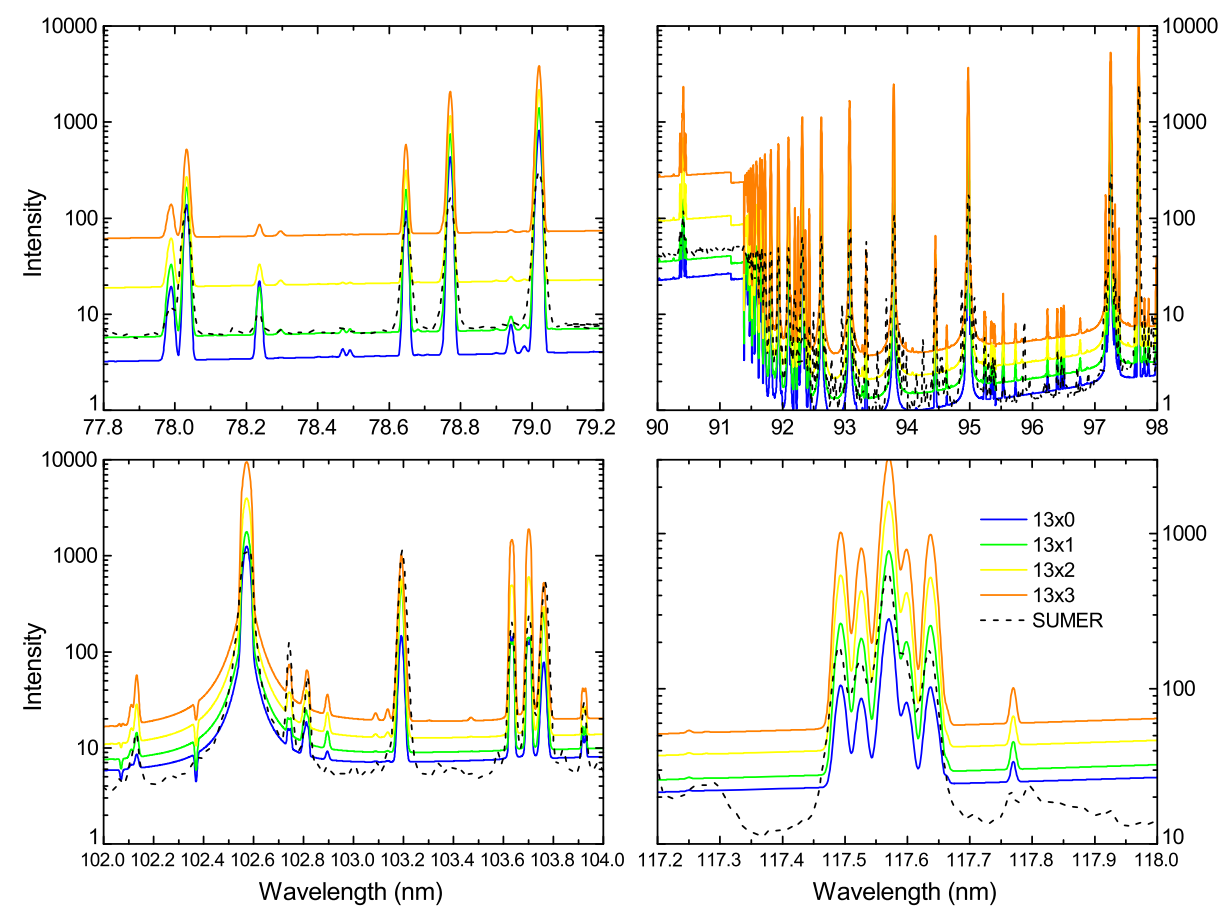

Figure 7 Top-left: the spectral range 77.8-79.2 nm showing Ne VIII 78.032, S v 78.647, and O IV 78.771 and $79.020 \mathrm{~nm}$ lines. Top-right: the range $90.0-98.0 \mathrm{~nm}$ showing the Ly continuum, Ly lines, and the prominent C III $97.702 \mathrm{~nm}$ line. Bottom-left: the $102-104 \mathrm{~nm}$ showing Ly $\beta$ at $102.572 \mathrm{~nm}$, O VI 103.191 and $103.761 \mathrm{~nm}$, and several C II lines. Bottom-right: the range $117.2-118.0 \mathrm{~nm}$ showing a number of C III lines.

electron conduction by Spitzer's formula); see also Article 3. Looking at Figure 1, we see that the C IV lines are formed at the temperature where the flux limiting starts to produce its steepening effect on the transition-region temperature.

Figure 7 shows other key lines covered by the SUMER spectrum. The O IV lines in the top-left panel are slightly overestimated with respect to SUMER (but much less overestimated than they were in Article 3). An increase in the flux-limiting coefficient would further reduce these lines' intensities, but it would lead to underestimating other lines from similar temperature ranges. Thus, the issue of O IV is still not optimally solved, and we note that $\mathrm{O}$ IV forms within a narrow temperature range and its ionization equilibrium can be easily affected by velocities.

Figure 7 (top-right) shows the head of the Ly continuum, the high Ly lines, and many other lines. The head of the Ly continuum gap in Article 3 is now fixed by the extension of the Ly continuum described in Section 5 and is very well reproduced by our current calculations. However, the high Ly lines are somewhat overestimated by the present calculations and this may be due to an over-simplification in our H-diffusion calculations (taken from FAL 1). In that procedure the diffusion velocity of excited $\mathrm{H}$ atoms is made equal to that of $\mathrm{H}$ lower levels, and this may not be correct when the levels are close to the continuum but a complicated revision to that procedure may require that each level's transition rates be considered to determine each excited-level diffusion velocity. The large intensity optically thick lines Ly $\gamma$ at $97.254 \mathrm{~nm}$ and C III $97.702 \mathrm{~nm}$ line are well reproduced by our calculations. 
Figure 8 The region near disk center averaged to create the EIS spectral atlas. The image coordinates are in arcsec with respect to disk center and has been created using the $\log$ intensity of the Fe XII $19.512 \mathrm{~nm}$ line. Black stripes indicate missing data and have not been used in the calculation of the EIS average spectrum.

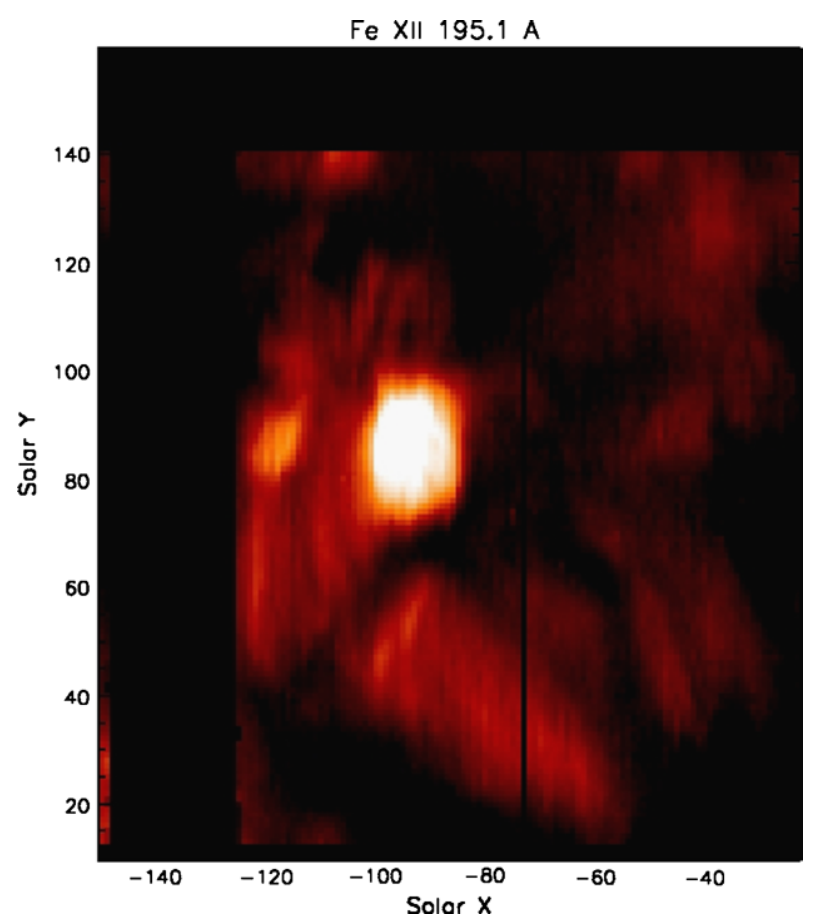

The optically thick Ly $\beta$ line and other strong lines shown in the bottom-left panel are also well reproduced despite the C I continuum being overestimated as indicated in Section 5. This continuum may have affected the peak intensities of some weaker optically thin lines, whose peak intensities are underestimated. Also, the Ly $\beta$ line wings may be affected by the C I continuum.

\subsection{Comparison of Selected Lines with Hinode/EIS}

The EIS spectrum was taken on 17 December 2008, and consisted of a $128^{\prime \prime} \times 128^{\prime \prime}$ raster of the quiet Sun near disk center. The entire wavelength range covered by EIS was transmitted to the ground. The exposure time was 90 seconds at each slit position. The data have been reduced, calibrated, and cleaned from cosmic rays using the standard EIS software available in SolarSoft. Figure 8 shows the field of view including a bright point and some faint structures. The dark stripes in this figure correspond to missing data that have not been considered. The emission from all pixels with non-zero intensity was averaged together to increase the signal-to-noise.

For this comparison, we convolve the computed spectra with a $\cos ^{2}$ bandpass of $5.5 \mathrm{pm}$ (i.e. $55 \mathrm{~mA}$ ) FWHM. From the present comparison it is apparent that the EIS data may be biased toward the lower temperature areas (model 13x0) than model $13 \times 1$ in our set of models. The $13 \times 3$ model perhaps corresponds to the bright point in this image.

Figure 9 shows two important lines that have also been targeted by imaging of the solar disk (e.g. by SDO/AIA, SOHO/EIT, and STEREO/EUVI). On the left panel, the Fe X line has relatively small changes between models $13 \times 0,13 \times 1$, and $13 \times 2$, i.e. the supergranular cell models of weak Ca II emission (see Article 3 and Table 1 in the present article). Therefore, the emission from all these models is close to the observed. However, on the 

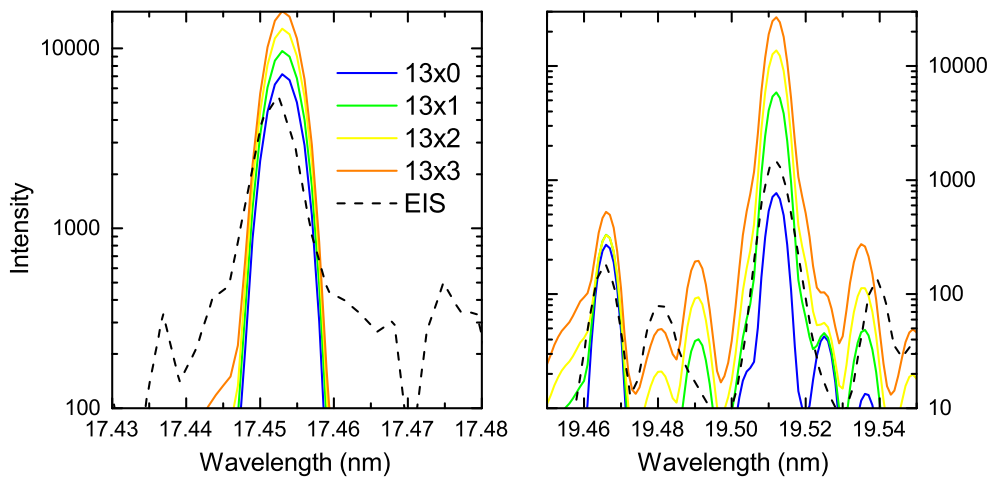

Figure 9 Left panel: the Fe X $17.453 \mathrm{~nm}$ line. Right panel: the Fe XI $19.512 \mathrm{~nm}$ and other lines including Fe VIII $19.466 \mathrm{~nm}$. Contamination by overlapping diffraction orders is shown at the shorter wavelengths and low intensities are not relevant.

right panel, the Fe XII line shows much larger model sensitivity and the EIS observation is slightly higher than the model $13 \mathrm{x} 0$ (the weakest of the quiet-Sun models) and smaller than the model $13 \times 1$. We note that the maximum temperature in model $13 \times 0$ barely surpasses $1 \mathrm{MK}$, see Figure 3, while model $13 \times 1$ reaches $1.4 \mathrm{MK}$. Also in the right panel of Figure 9 is the Fe VIII $19.466 \mathrm{~nm}$ line, which shows little model sensitivity and is only slightly overestimated by our models. Other structure in the right panel is a line at $19.48 \mathrm{~nm}$ that is apparent in the EIS data but computed to be very weak in our models of quiet-Sun network from a blend of Fe IX, X, and XI lines with high excitation lower levels. In addition, we have a computed line at around $19.49 \mathrm{~nm}$, which results from a blend of Fe XII 19.490 and Ni X $19.491 \mathrm{~nm}$ but is not clearly shown in EIS data.

Figure 10 shows many lines spanning from upper transition-region temperatures to the hot Fe XIII, and including the low transition-region He II $25.632 \mathrm{~nm}$ line. In the Fe XIII lines the contrast between our models $13 \times 0$ to $13 \times 3$ is rather large and model $13 \times 0$ is always closest to the EIS intensities. In the transition-region lines the contrast between $13 \times 0$, $13 \times 1$, and $13 \times 2$ is small, and the emission from these three models are all close to the EIS observations. For the He II $25.6 \mathrm{~nm}$ line, the EIS observation falls closer to $13 \times 1$ but $13 \times 0$ emission is not very different. In this figure we also note cases where lines are computed but not observed, and overall in the spectrum we also see EIS lines that do not show a computed equivalent line. We note that in some cases, especially for faint lines, the lines' wavelengths used were the theoretical ones in CHIANTI 7.1 data and may not be accurate, while in the cases where observed wavelengths are available we have used these.

\subsection{Comparison with SOHO/CDS}

The CDS instrument observed the solar spectrum in six spectral windows between 15.0 and $78.5 \mathrm{~nm}$ using two different spectrometers: the Normal Incidence Spectrometer (NIS), observing in the 30.7-37.9 $\mathrm{nm}$ and 51.3-63.3 nm ranges, and the Grazing Incidence Spectrometer (GIS), observing in the $15.1-22.1 \mathrm{~nm}, 25.6-34.1 \mathrm{~nm}, 39.3-49.2 \mathrm{~nm}$, and 65.9$78.5 \mathrm{~nm}$ ranges. Due to the presence of ghost lines in the GIS spectrum, we only considered the NIS spectral ranges. The observation that we used to determine the quiet-Sun spectral atlas was taken on 6 October 1996, during solar minimum, at solar center, and was part of the standard NIS spectral-atlas program: NISAT. The NIS $2^{\prime \prime} \times 240^{\prime \prime}$ slit was used at ten ad- 

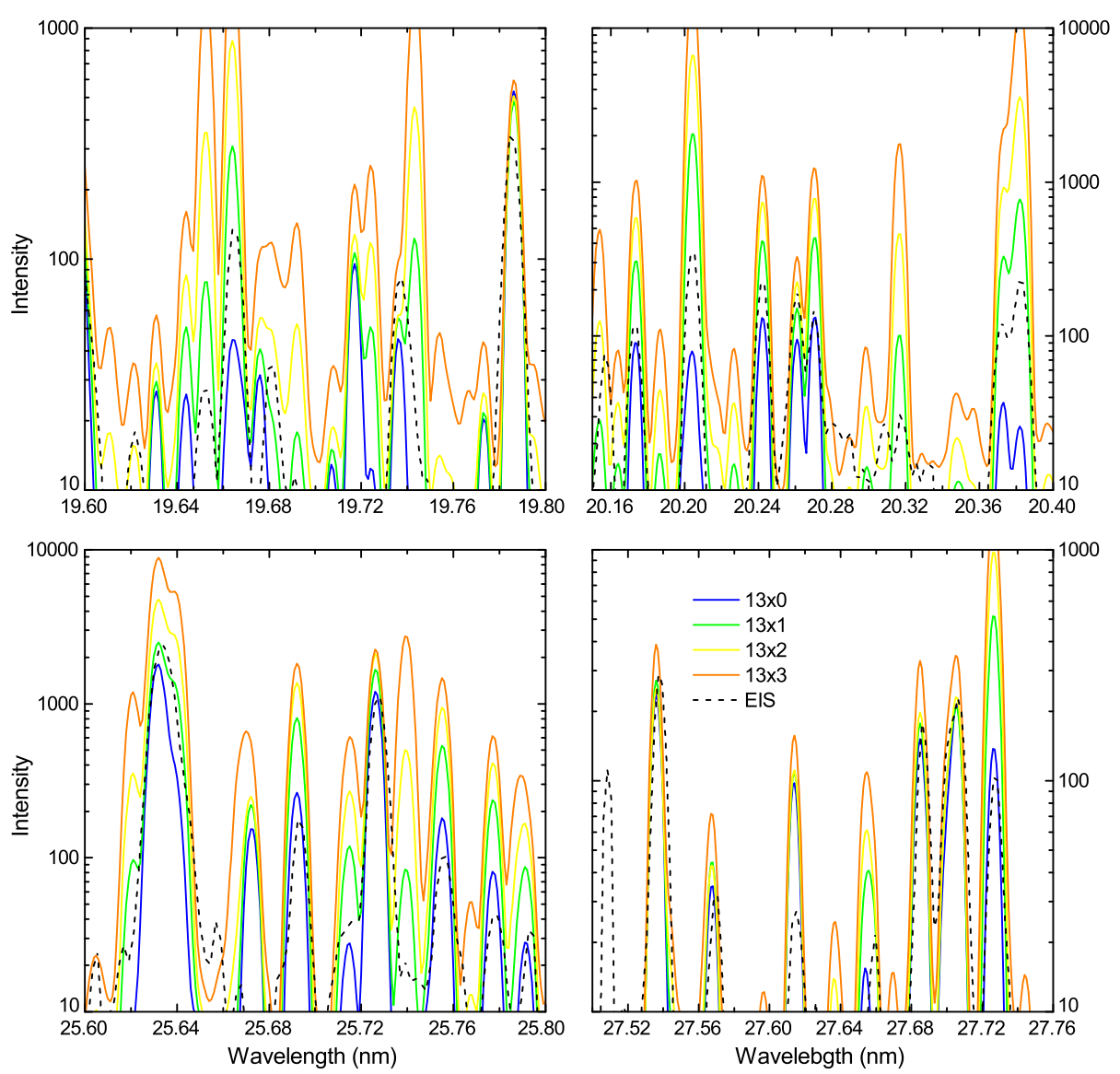

Figure 10 Top-left: the range 19.6-19.8 nm showing Fe XIII 19.652, Fe XIII 19.743, and Fe IX 19.786 nm lines. Top-right: the range 20.16-20.4 nm showing the Fe XII 20.174, Fe XIII 20.204, Fe XI 20.242, Fe XII 20.373, and Fe XIII $20.382 \mathrm{~nm}$ lines among others. Bottom-left: the range 25.6-25.8 nm showing He II 25.632, Fe XI 25.692, Fe X 25.726, Fe XI 25.755, and Fe XI $25.777 \mathrm{~nm}$ lines. Bottom-right: the range 27.5-27.8 nm showing Si VII 25.736, Mg VII 27.615, Mg V 27.658, Si VII/VIII 27.685, Mg VII/Si VIII 27.704, and Si X $27.726 \mathrm{~nm}$ lines.

jacent positions along the E-W direction, for a total field of view of $20^{\prime \prime} \times 240^{\prime \prime}$. At each slit position, the solar spectrum was observed for 50 seconds. The entire field of view was averaged together in order to increase the signal-to-noise ratio. For this comparison we convolve the computed spectra with a $\cos ^{2}$ bandpass of $55 \mathrm{pm}$ (i.e. $550 \mathrm{~mA}$ ) FWHM.

In this section we show only a couple of lines, but from these and other comparisons it seems apparent that the SOHO/CDS "quiet-Sun" data were selected, much like the SOHO/SUMER data, toward the feature we represent by $13 \times 1$. Therefore, Figure 11 only compares with the disk-center spectrum from this model.

Figure 11 shows that the $\mathrm{O}$ IV lines computed using model 13x1 are still somewhat larger than the observed by CDS, but the O v computed line matches the observed well. It is likely that the simple flux limiting that we used needs improvement probably in its temperature or density dependence. However, there may be other reasons for this discrepancy, e.g. O IV ionization treatment may need improvement. 

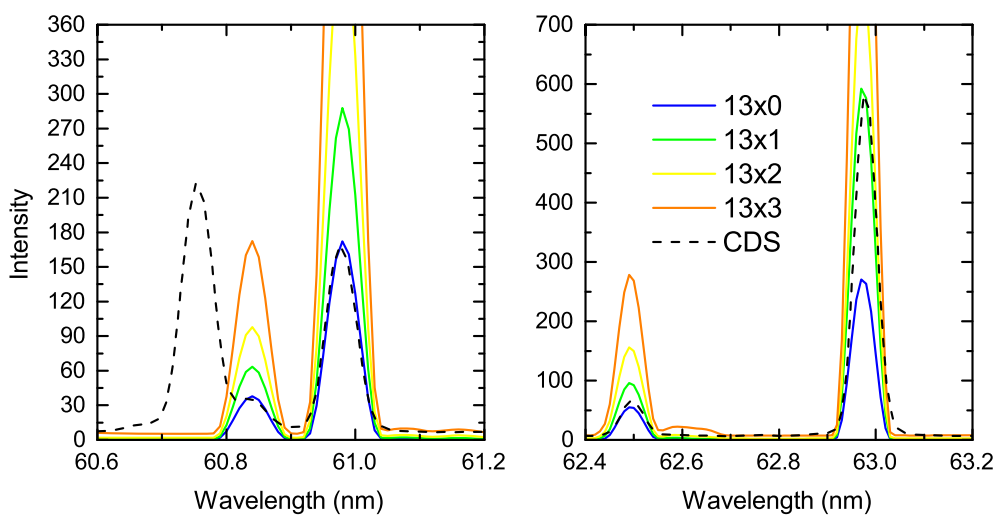

Figure 11 Left: the spectral range 60.8 -61.2 nm showing O IV 60.840 and $60.983 \mathrm{~nm}$ lines. The spectral line shown at $\approx 60.75 \mathrm{~nm}$ is actually the He II $30.378 \mathrm{~nm}$ from the second-order diffraction. Right: the range $62.4-63.2 \mathrm{~nm}$ showing O V $62.973 \mathrm{~nm}$ line.

\section{Comparison with Solar Spectral Irradiance Observations}

The comparison of the complete SSI with model $13 x 1$ was shown above in Figure 6 , and the continua issues were discussed in Section 5. In the present section we discuss some details of the SSI that cannot be addressed by the graph in Figure 4 but need more detail on the lines.

For comparing the quiet-Sun EUV irradiance spectrum, we use the SDO/EVE rocket spectrum taken in April 2008 during a very quiet period, near the solar-activity minimum; however, on the day that the spectrum was taken a small active region was present on the disk and also coronal holes extended into low-latitude areas. The EVE instrument was calibrated before and after this rocket flight on beam line 2 (BL2) at the Synchrotron Ultraviolet Radiation Facility III (SURF-III) located in the National Institute of Standards and Technology (NIST) in Gaithersburg, Maryland, USA (see Chamberlin et al., 2009 and also Woods et al., 2009). We also use the SORCE/SOLSTICE spectrum taken in April 2005, which, although not at solar minimum, corresponded to fairly quiet conditions in terms of the chromospheric features observed on the disk. This instrument calibration was maintained in flight through comparisons with relatively bright stars that are believed to be constant (see Snow et al., 2005, and McClintock, Snow, and Woods, 2005).

The highest resolution that was achieved by these high-quality and comprehensive SSI data was $0.1 \mathrm{~nm}$. Although we do not know in detail the bandpass shape of the SSI instruments, we base our present comparison on a nominal resolution of $0.1 \mathrm{~nm}$ FWHM. Therefore we convolve our computed SSI for all our models with this profile using again a $\cos ^{2}$ filter.

As was noted in Article 3, the Ca II K images show that the features corresponding to the chromospheric quiet Sun are more or less uniformly spread over the solar disk. The same is not quite true for the more recent images from SDO/AIA that show, in the $160 \mathrm{~nm}$ images, areas of depleted or increased network components that may relate to the growth and decay of active regions. However, this topic is beyond the present article and here we will show the irradiance that would be produced if the entire disk were covered by models $13 \times 0,13 \times 1,13 \times 2$, or $13 \times 3$. If the whole disk were covered by a uniform distribution of these features then the SSI can be calculated by a weighted sum of these components SSI. This corresponds to assuming the weights as independent of the disk position. 

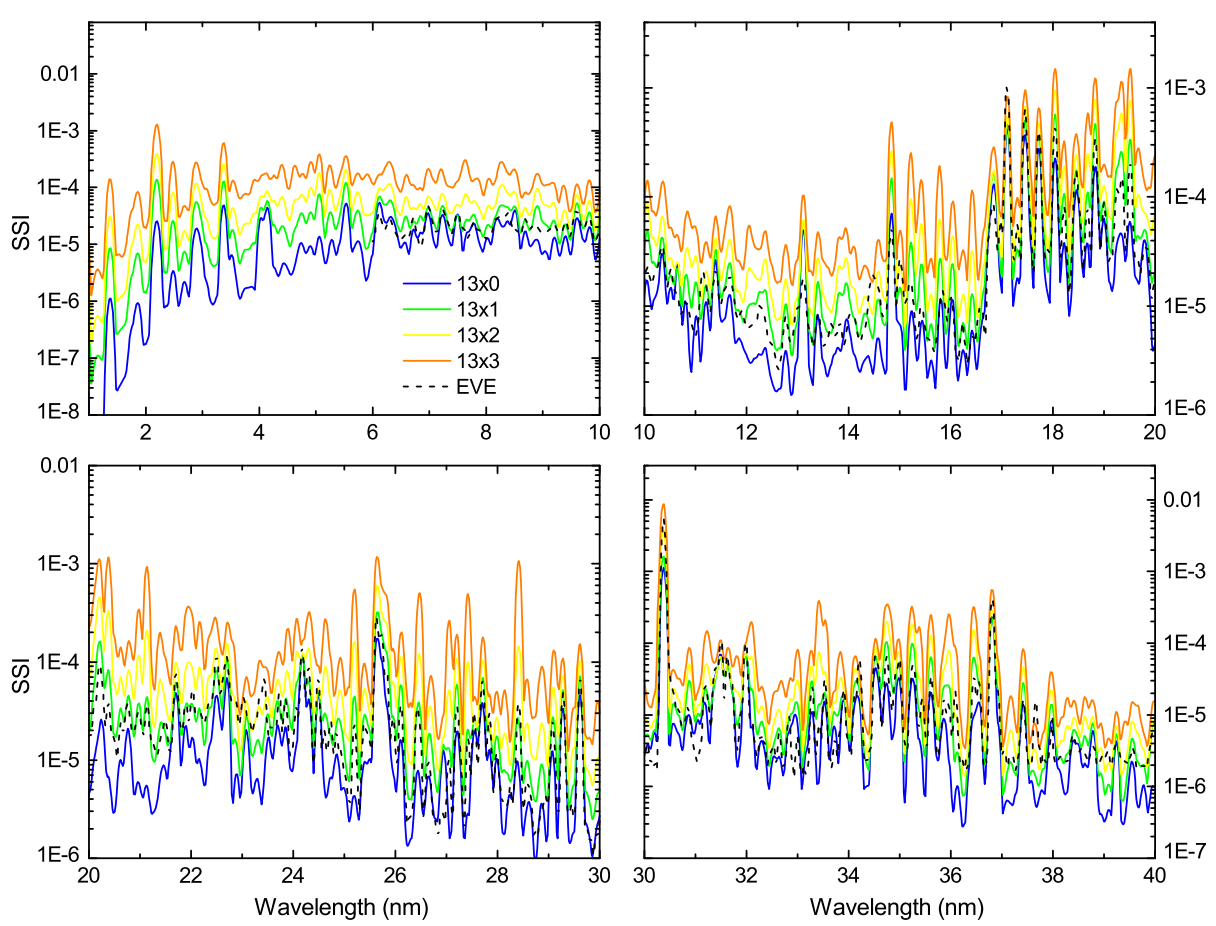

Figure 12 Comparisons of the SSI from the various components of the quiet Sun with the SSI observed by $\mathrm{SDO} / \mathrm{EVE}$ in the range $1-40 \mathrm{~nm}$.

The above assumption may not be as good for the coronal features because the presence of coronal holes is predominant over the polar high-latitude regions. However, this issue is beyond the scope of the present article and is related to assigning the weights of the various features as dependent on the position on the solar disk using images.

Figures 12 and 13 show the good agreement between the results that we obtain for an imaginary solar disk composed only of model $13 \times 1$ and the SDO/EVE measurements at a time when solar activity was near minimum. The tracings for the imaginary disk entirely composed of the other quiet-Sun component models show curves generally above and below the model 13x1 and probably compensate, but this is beyond the current article because the determination of the corresponding weights depends on the images of the solar disk.

Figure 14 last panel shows the issue with the continuum that was discussed in Section 5, and also two emission lines: an unresolved complex of C I lines around $165.8 \mathrm{~nm}$ and $\mathrm{Al} \mathrm{II}$ $167.078 \mathrm{~nm}$, whose computed irradiances are much larger than what is observed. We already mentioned the issue of C I in Section 5. The atomic data available for Al II are very old (from Aggarwal and Keenan, 1994) and may not be sufficiently accurate. These lines are examples of how critical for the present work is the availability of accurate collisional excitation rates.

\section{Effects of Solar Activity on the SSI}

In this section we show some comparisons with observed SSI in order to assess the quality of our models for estimating the SSI variability over the relatively short-term periods of rotational modulation, i.e. the variation of the SSI due to the non-uniformity of the distribution 

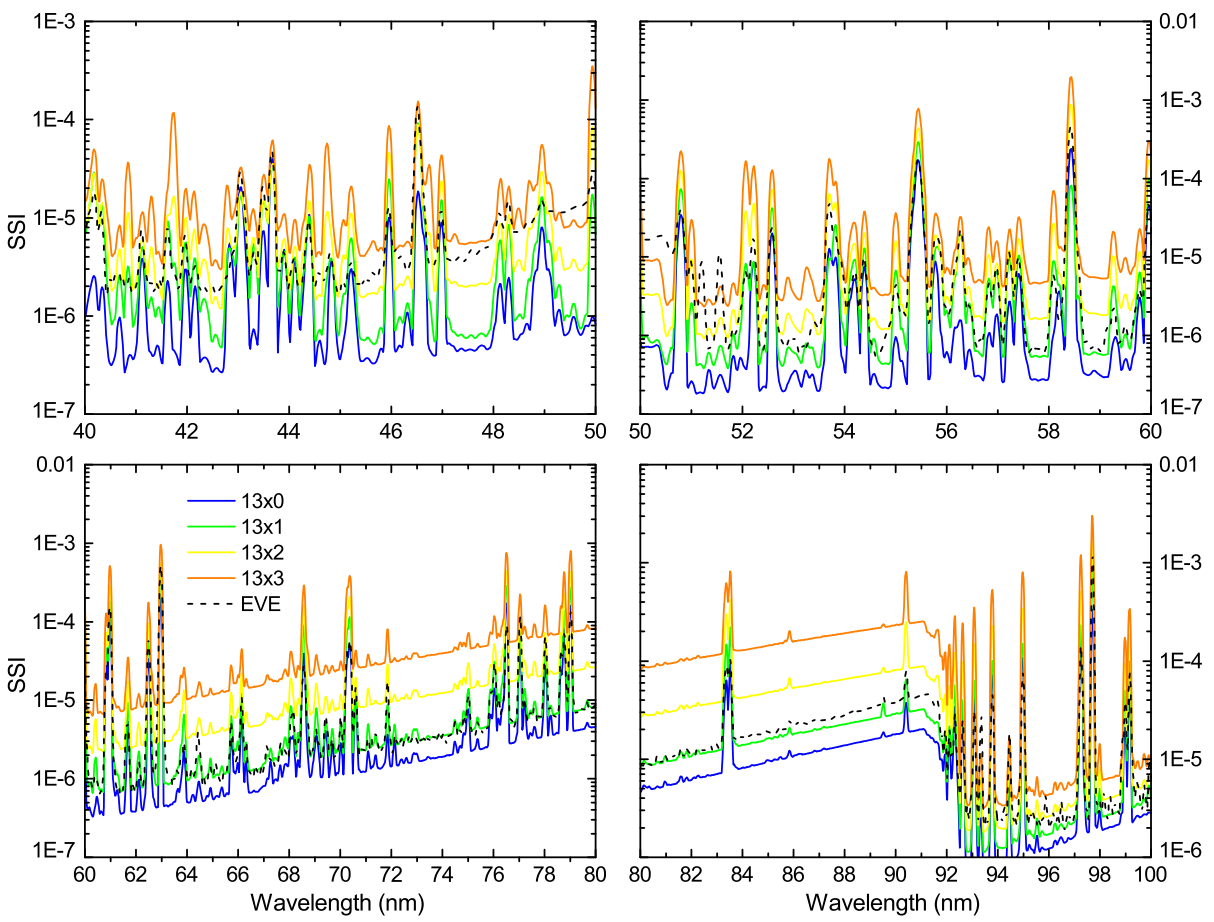

Figure 13 Comparisons of the SSI from the various components of the quiet-Sun with the SSI observed by SDO/EVE in the range $40-100 \mathrm{~nm}$.

of active regions over the solar surface and their transiting of the solar disk as a consequence of the solar rotation.

It is impossible to separate the SSI variability from the images used to determine which features are present in each part of the solar disk. For our calculation it is only necessary to know which are the relative areas occupied by each feature at a given heliocentric angle $[\theta]$ or equivalently at each $\mu=\cos (\theta)$. These relative areas are determined, in our method, by a combination of images and $\mu$-dependent thresholds. Some discussion on the image processing was given in Article 3, a detailed discussion of our method new sources for determining the relative areas, or weights, is beyond the scope of this article. So far we use only one image per day of each type and therefore intra-day variations on the solar surface or on the image quality may affect our measurements. Also, we note that we currently use two masks of features, one for the chromospheric/lower-transition-region and the other for the upper-transition-region/corona. The data that we present here are based on SDO/AIA images and essentially uses the 160 and $19.3 \mathrm{~nm}$ images for the chromosphere, and the $9.4 \mathrm{~nm}$ images for the corona. In this way, the coronal and the chromospheric SSI components are determined separately and the two components are added to form the total SSI, since we have verified that the coronal component is optically thin and effects on the SSI of absorption of the chromospheric component are negligible.

Figure 15 shows the comparison between the computed and observed SSI variation of the peak of a rotational modulation and its bottom, relative to the latter. The peak occurred on 23 October 2012 and for determining the bottom we averaged the two nearby minima on 9 October 2012 and 5 November 2012. Again we stress that the EVE data in this comparison 

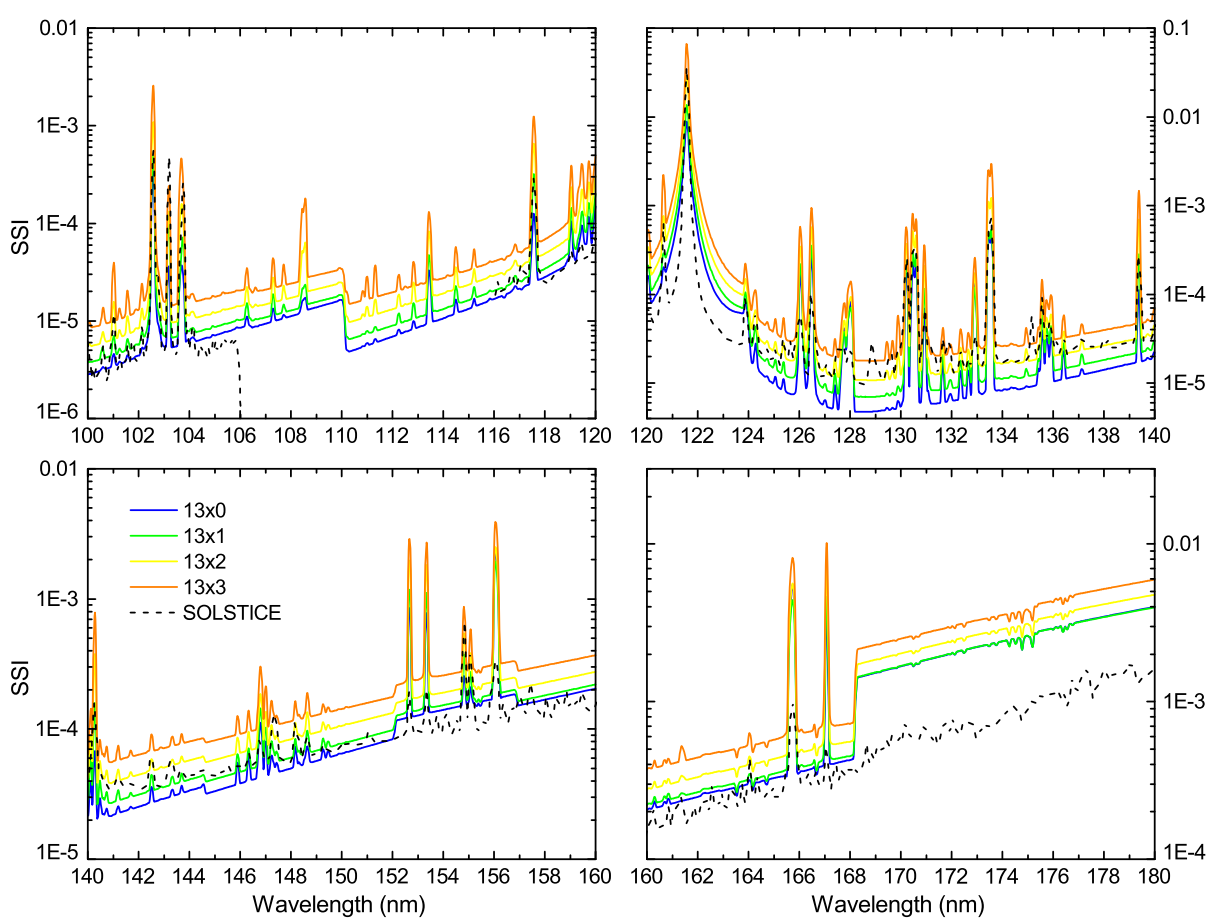

Figure 14 Comparisons of the SSI from the various components of the quiet Sun with the SSI observed by SORCE/SOLSTICE in the range $100-180 \mathrm{~nm}$.

Figure 15 Comparison of the SSI relative variation between the top and bottom of a rotational modulation in October 2012.

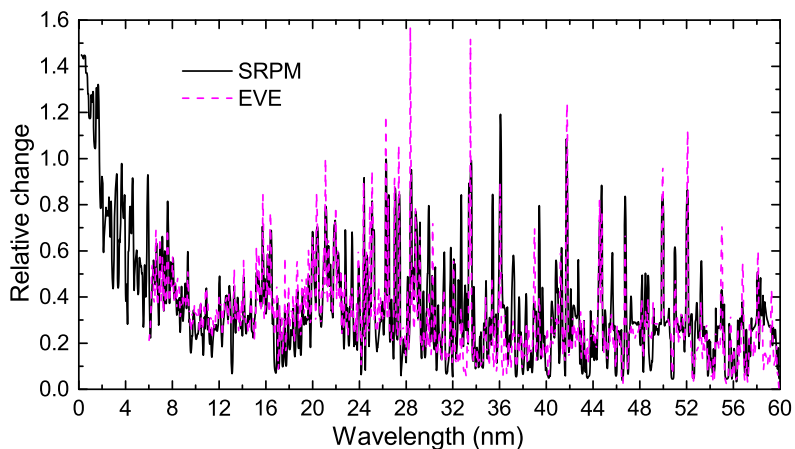

corresponded to a daily average but the SRPM computed data corresponds to single-image snapshots from SDO/AIA using each filter. The figure shows fairly good agreement but the relative change is slightly underestimated in some lines by SRPM. A closer inspection showed that some of these differences occur because of the EVE data in those cases have a slightly higher spectral resolution than the $0.1 \mathrm{~nm} \mathrm{FWHM} \cos ^{2}$ filter used to degrade the computed spectra. However, we believe that the variations in the lines near 28 and $34 \mathrm{~nm}$ were truly underestimated. These largest peaks in the relative variation correspond to the well-known Fe XV 28.3163 and Fe XVI 33.541 nm lines, which show very large enhancements in flares, and probably it is due to small flares that are included in the EVE daily average. 
Figure 16 The SSI relative variation between the top and bottom of a rotational modulation in October 2012, at long wavelengths in the EUV.

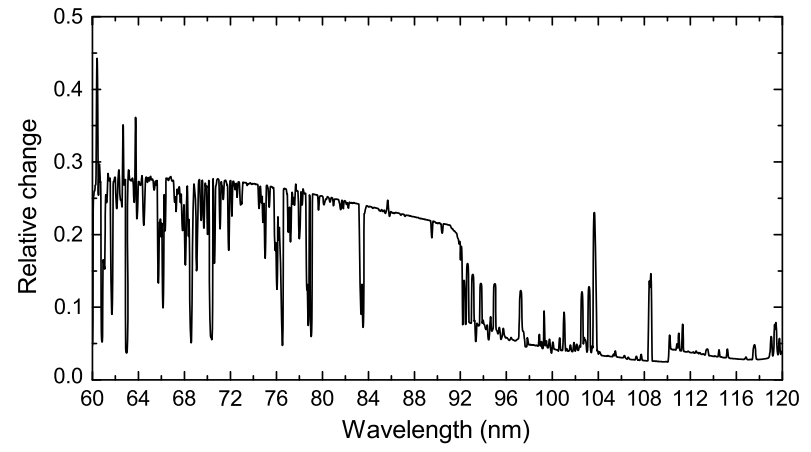

Figure 17 Comparison of the FUV SSI relative variation between the top and bottom of a rotational modulation, i.e. relative increase on 23 October with respect to 9 October 2012 .

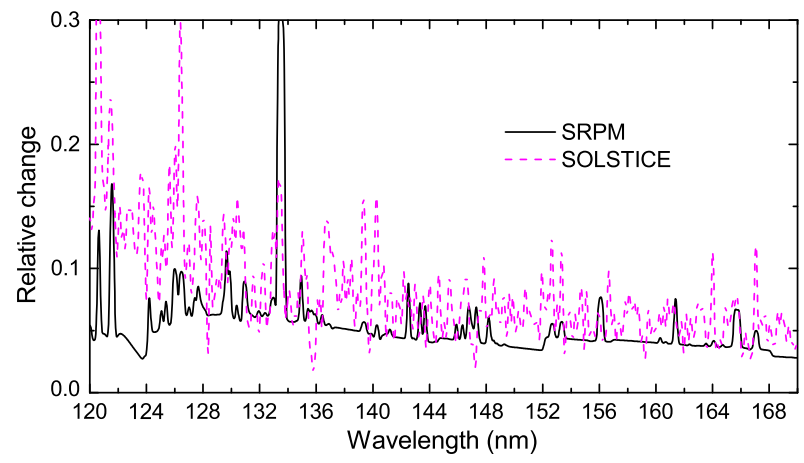

Also, note the large increase in the variability at short wavelengths and even at the smallest that we include in this graph, namely $0.22 \mathrm{~nm}$, shorter than EVE can observe. This variability at the very short wavelengths is comparable to the largest variability EVE observed at the lines near 28 and $34 \mathrm{~nm}$, but our calculations does not include flares. When flares are included, the variations at very short wavelengths are probably larger than those shown in Figure 15 and could have significant effects on photoelectrons produced in the Earth's upper atmosphere, see Peterson et al. (2012).

The EVE peak in the rotational modulation spectra seems to occur at a fraction of a tenth of a nm (i.e. fraction of an $\AA$ ) shorter wavelengths in many lines; this shift maybe due to wavelength calibration uncertainty. Also, we note that our calculations give an even larger relative variation at wavelengths shorter than those shown in Figure 15. This figure could not include the shorter wavelengths because of the convolution imposed.

For comparisons at longer wavelengths, we need to rely on other data because the EVE version 3 that we have used does not yet include reliable degradation calibration at wavelengths longer than those shown here. An update of the EVE calibration for the year 2012 is forthcoming and will improve the longer wavelengths. Figure 16 shows the computed SSI variation in the range $60-120 \mathrm{~nm}$, and it is interesting to note that the SSI in the Ly continuum shows an important variation due to rotational modulation in our calculations, and it also shows that its maximum amplitude does not occur at the head of this continuum.

Figure 17 shows the comparison of the computed rotational modulation with the SORCE/SOLSTICE data. For this comparison there were no observations on 5 November 2012, and the comparison is of the variation between the two days in October. Again here the SOLSTICE data correspond to a daily average. Although the original data from Level 2 processing is at $0.1 \mathrm{~nm}$ resolution we had to smooth the data at $0.2 \mathrm{~nm}$ resolution to reduce 
the noise. Of course we applied the same smoothing for the SRPM data; however, we note that effectively there is no noise in the computed data (only much lower numerical noise exists).

Figure 18 shows the masks used for the corona/upper-transition-region and the chromosphere/lower-transition-region in the computation of the total SSI. Note that although on the peak of the rotational modulation there are more hot active regions, at the minimum of the rotation plage are still present on the solar disk although in less total area than at the maximum of the rotational modulation.

\section{Discussion}

We have shown a set of physical models and comparisons between the computed and observed spectra that show that a semi-empirical set of models can account for the observed EUV SSI and its variation with solar activity. We consider the overall agreement good although in some details it can be improved by further work on atomic data, especially collision rates, minor improvements to the physical models, and perhaps a more complete physical description containing more components. However, it should be kept in mind that including more components would only improve the description of solar activity if there were a way to determine daily (or more frequently) the mix of components from solar images, and if their radiative interaction were either negligible or accounted for. Otherwise, a description with more components would not necessarily improve the results over those from the present set of models.

Moreover, because one of the important applications of the present research is in the forecasting of the EUV SSI, if more components were included it would also be necessary to forecast the expected evolution of the mix of components. This poses a question for the current set of features that Fontenla et al. (2009b) addressed using data from the farside of the Sun. Although these techniques have produced good results for Ly $\alpha$, their forecasting power for other spectral regions needs to be further explored, and it would likely not be as precise unless EUV data from the farside is available because otherwise inference of the coronal features must rely on correlations with Ly $\alpha$. For the present, STEREO/EUVI data provide additional input because the spacecraft observes the farside of the Sun, but in the long term the positions of the spacecraft or instrument failure will make their observations irrelevant in forecasting. We suggest that further consideration be given to keeping and improving ways for imaging the farside at all wavelengths possible and using a variety of techniques.

Also, more theoretical considerations of the physical mechanisms that produce coronal and chromospheric heating by magnetic fields would greatly enhance the forecasting power of even limited observations of the farside. If the evolution of magnetic-field structures could be forecast, then the connection with SSI requires the ability to infer from the magnetic field which coronal and chromospheric heating enhancements would result and which would be the spectra emitted by these magnetic features. This article shows that when the physical characteristics of the various features in the solar atmosphere are known, even in a highly simplified manner, it is possible to produce SSI spectra that match the observations fairly well.

The results presented here encourage the use of stellar models including chromospheres and coronae for the investigation of stars other than the Sun. Such studies yield important information that impacts the planets around such stars, i.e. "exoplanets", and can produce 

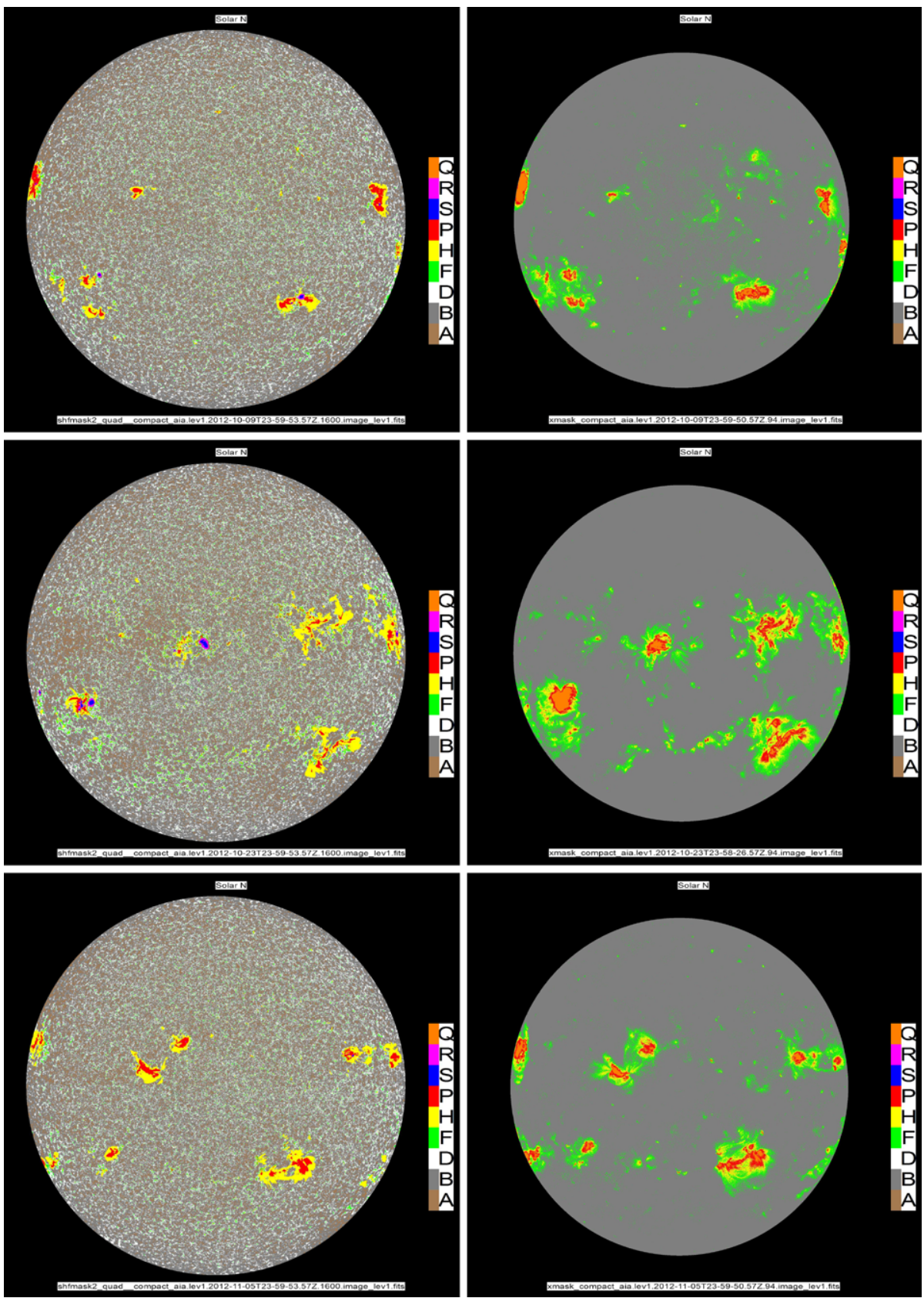

Figure 18 Image masks showing the features detected in the solar disk according to our classification in Table 1 and the SDO/AIA images. The left-side panels show the chromospheric masks, the right-side panels show the coronal masks. The corresponding dates are from top to bottom, 9 October, 23 October, and 5 November 2012. 
important information about the EUV/FUV environment of such planets. For instance, Linsky et al. (2011) have shown that solar models with increasing heating rates fit stellar FUV continua $(115.8-149.2 \mathrm{~nm})$ for $\mathrm{G}$ stars with increasing heating rates.

Acknowledgements The work by JF was funded mainly by LWS-NASA grant NNX09AJ22G. Also, AF grant FA9453-13-M-0063 funded in a small part the work by JF, EL, and TW. We acknowledge the NIST Database that was funded (in part) by the Office of Fusion Energy Sciences of the US Department of Energy, by the National Aeronautics and Space Administration, by NIST's Standard Reference Data Program (SRDP), and by NIST's Systems Integration for Manufacturing Applications (SIMA) Program. We also acknowledge the CHIANTI team for providing atomic data that made this work possible. CHIANTI is a collaborative project involving George Mason University, the University of Michigan (USA) and the University of Cambridge (UK). Hinode is a Japanese mission developed and launched by ISAS/JAXA, with NAOJ as domestic partner and NASA and STFC (UK) as international partners. It is operated by these agencies in cooperation with ESA and NSC (Norway). Data are provided by courtesy of NASA SDO/AIA and EVE science teams.

Open Access This article is distributed under the terms of the Creative Commons Attribution License which permits any use, distribution, and reproduction in any medium, provided the original author(s) and the source are credited.

\section{References}

Avrett, E.H., Loeser, R.: 2008, Astrophys. J. Suppl. 175, 229.

Aggarwal, K.M., Keenan, F.P.: 1994, J. Phys. B 27, 5321.

Bale, S.D., Pulupa, M., Salem, C., Chen, C.H.K., Quataert, E.: 2013, Astrophys. J. Lett. 769, L2.

Brysk, H., Campbell, P.M., Hammerling, P.: 1975, Plasma Phys. 17, 473.

Catto, P.J., Grinneback, M.: 2000, Phys. Lett. A 277, 323.

Chamberlin, P.C., Woods, T.N., Crotser, D.A., Eparvier, F.G., Hock, R.A., Woodraska, D.L.: 2009, Geophys. Res. Lett. 36, L05102. doi:10.1029/2008GL037145.

Culhane, J.L., Harra, L.K., James, A.M., Al-Janabi, K., Bradley, L.J., Chaudry, R.A., et al.: 2007, Solar Phys. 243, 19. doi:10.1007/s01007-007-0293-1.

Curdt, W., Brekke, P., Feldman, U., Wilhelm, K., Dwivedi, B.N., Schühle, U., Lemaire, P.: 2001, Astron. Astrophys. 375, 591.

Fontenla, J.M., Avrett, E.H., Loeser, R.: 1990, Astrophys. J. 355, 700.

Fontenla, J.M., Avrett, E.H., Loeser, R.: 1991, Astrophys. J. 377, 712.

Fontenla, J.M., Avrett, E.H., Loeser, R.: 1993, Astrophys. J. 406, 319.

Fontenla, J.M., Balasubramaniam, K.S., Harder, J.: 2007, Astrophys. J. 667, 1243.

Fontenla, J.M., White, O.R., Fox, P.A., Avrett, E.H., Kurucz, R.L.: 1999, Astrophys. J. 518, 480.

Fontenla, J.M., Curdt, W., Haberreiter, M., Harder, J., Tian, H.: 2009a, Astrophys. J. 707, 482.

Fontenla, J.M., Quémerais, E., González Hernández, I., Lindsey, C., Haberreiter, M.: 2009b, Adv. Space Res. 44, 457.

Fontenla, J.M., Harder, J., Livingston, W., Snow, M., Woods, T.: 2011, J. Geophys. Res., Atmos. 116, D20108. doi:10.1029/2011JD016032.

Harrison, R.A., Sawyer, E.C., Carter, M.K., Cruise, A.M., Cutler, R.M., Fludra, A., et al.: 1995, Solar Phys. 162, 233. ADS:1995SoPh..162..233H, doi:10.1007/BF00733431.

Kramida, A., Ralchenko, Yu., Reader, J. (NIST ASD Team): 2013, NIST Atomic Spectra Database (ver. 5.0), http://physics.nist.gov/asd. Cited April 2013. National Institute of Standards and Technology.

Landi, E., Young, P.R., Dere, K.P., Del Zanna, G., Mason, H.E.: 2013, Astrophys. J. 768, 94.

Linsky, J.L., Bushinsky, R., Ayres, T., Fontenla, J., France, K.: 2011, Astrophys. J. 745, 25.

MacNeice, P., Fontenla, J.M., Ljepojevic, N.N.: 1991, Astrophys. J. 369, 544.

McClintock, W.E., Snow, M., Woods, T.N.: 2005, Solar Phys. 230, 259. ADS:2005SoPh..230..259M, doi: 10.1007/s11207-005-1585-5.

Peterson, W.K., Woods, T.N., Fontenla, J.M., Richards, P.G., Chamberlin, P.C., Solomon, S.C., Tobiska, W.K., Warren, H.P.: 2012, J. Geophys. Res., Atmos. 117, A05320. doi:10.1029/2011JA017382.

Rosner, R., Tucker, W.H., Vaiana, G.S.: 1978, Astrophys. J. 220, 643.

Reale, F.: 2010, Living Rev. Solar Phys. 7, 5. doi:10.12942//rsp-2010-5.

Schurtz, G.P., Nicolai, D., Busquet, M.: 2000, Phys. Plasmas 7, 4328.

Seaton, M.J.: 1962, Proc. Phys. Soc. 79, 1105.

Seaton, M.J.: 1987, J. Phys. B, At. Mol. Phys. 20, 6363S. doi:10.1088/0022-3700/20/23/026. 
Serio, S., Peres, G., Vaiana, G.S., Golub, L., Rosner, R.: 1981, Astrophys. J. 243, 288.

Snow, M., McClintock, W.E., Rottman, G.J., Woods, T.N.: 2005, Solar Phys. 230, 295. ADS:2005SoPh.. 230..295S, doi:10.1007/s11207-005-8763-3.

Spitzer, L. Jr.: 1962, Physics of Fully Ionized Plasmas, Interscience, New York.

Wang, Y., Zatsarinny, O., Bartschat, K.: 2013, Phys. Rev. A 87, 012704.

Wilhelm, K., Curdt, W., Marsch, E., Schühle, U., Lemaire, P., Gabriel, A., et al.: 1995, Solar Phys. $162,189$. ADS:1995SoPh..162..189W, doi:10.1007/BF00733430.

Woods, T.N., Chamberlin, P.C., Harder, J.W., Hock, R.A., Snow, M., Eparvier, F.G., Fontenla, J., McClintock, W.E., Richard, E.C.: 2009, Geophys. Res. Lett. 36, L01101. doi:10.1029/2008GL036373.

Wyndham, E.S., Kilkenny, J.D., Chuaqui, H.H., Dymoke-Bradshaw, A.K.L.: 1982, J. Phys. D, Appl. Phys. 15, 1683 . 